\title{
Article \\ The Promising Mechanisms of Low Molecular Weight Compounds of Panax Ginseng C.A. Meyer in Alleviating COVID-19: A Network Pharmacology Analysis
}

\author{
Ki-Kwang Oh (D), Md. Adnan (D) and Dong-Ha Cho *
}

Citation: Oh, K.-K.; Adnan, M.; Cho, D.-H. The Promising Mechanisms of Low Molecular Weight Compounds of Panax Ginseng C.A. Meyer in Alleviating COVID-19: A Network Pharmacology Analysis. Processes 2022, 10, 333. https://doi.org/ $10.3390 /$ pr10020333

Academic Editor: Alina Pyka-Pająk

Received: 11 January 2022

Accepted: 7 February 2022

Published: 9 February 2022

Publisher's Note: MDPI stays neutral with regard to jurisdictional claims in published maps and institutional affiliations.

Copyright: (C) 2022 by the authors. Licensee MDPI, Basel, Switzerland. This article is an open access article distributed under the terms and conditions of the Creative Commons Attribution (CC BY) license (https:// creativecommons.org/licenses/by/ $4.0 /$ )
Department of Bio-Health Convergence, College of Biomedical Science, Kangwon National University, Chuncheon 24341, Korea; nivirna07@kangwon.ac.kr (K.-K.O.); mdadnan@kangwon.ac.kr (M.A.)

* Correspondence: chodh@kangwon.ac.kr; Tel.: +82-33-250-6475

\begin{abstract}
Panax Ginseng C.A. Meyer (PGCAM) is a well-known phytomedicine, but most of its compounds, such as ginsenoside derivatives, have poor absorption and bioavailability profile due to high molecular weight ( $\geq 500$ Daltons), which is the major hurdle for their clinical application. Hence, this research explored the efficiency of low molecular weight compounds (LMWCs) ( $<500$ Daltons) screened from PGCAM and their anti-COVID-19 mechanisms through network pharmacology. Molecular compounds from PGCAM were identified using public databases and filtered out by the drug-likeness evaluation. Genes interacted with these filtered compounds, and COVID-19-related genes were extracted from public databases. In addition, overlapping genes between compounds and interactive genes were identified using the Venn diagram. In parallel, the networking between compounds and overlapping genes was analyzed by RStudio. The pathway enrichment analysis of overlapping genes was determined by STRING. Finally, the key bioactive compounds were documented through virtual screening. The bubble chart suggested that the mechanisms of PGCAM against COVID-19 were related to 28 signaling pathways. The key molecular anti-COVID-19 mechanisms might be the anti-inflammation, anti-permeability, and pro-apoptosis by inactivating the PI3K-Akt signaling pathway. The six key genes and the five compounds related to the PI3K-Akt signaling pathway were RELA-paeonol, NFKB1-frutinone A, IL6-nepetin, MCL1-ramalic acid, VEGFA-trifolirhizin, and IL2-trifolirhizin. The docking between these key genes and compounds demonstrated promising binding affinity with a good binding score. Overall, our proposed LMWCs from PGCAM provide a fundamental basis with noteworthy pharmacological evidence to support the therapeutic efficacy of PGCAM in relieving the main symptoms of COVID-19.
\end{abstract}

Keywords: Panax Ginseng C.A. Meyer; low molecular weight compounds (<500 Daltons); COVID-19; PI3K-Akt signaling pathway; anti-inflammation and anti-permeability; pro-apoptosis

\section{Introduction}

Due to the intense efforts made by clinicians and researchers, only one human infection, "smallpox", has been successfully eradicated from the world [1]. However, the recent outburst of a new type of pneumonia caused by a severe acute respiratory syndrome coronavirus 2 (SARS-CoV-2) [2] is not likely to disappear from the globe completely, even after the quick development of a sustainable vaccine. This indicates one certain thing: that the world needs novel treatment plans and possible therapeutic interventions to combat the highly infectious disease (COVID-19), no matter what else happens next.

In late December 2019, a life-threatening contagious disease with unknown etiology was first reported in Wuhan, Hubei province, China [3]. Although infectious disease experts identified the new coronavirus (SARS-CoV-2) spread from the animal host, concerns remained over how the first person might have been infected [4]. Viral genomics and epidemiological studies evidenced that the animal-to-human shifted SARS-CoV-2 can rapidly transmit from one person to another through interaction or respiratory droplets $[5,6]$. 
Hence, the World Health Organization (WHO) initially declared a global emergency on 22 January 2020, as the Wuhan coronavirus spread. On 20 August 2020, the Center for Systems Science and Engineering (CSSE) at Johns Hopkins University reported a health crisis with intensifying global case numbers $(22,260,000)$ and fatalities [7,8]. COVID-19 predominantly attacks the respiratory system, even though other organ systems are compromised. Lower respiratory tract infection (LRTI) associated with fever, shortness of breath, and dry cough were reported as the symptoms with the first case from Wuhan, China [9]. Additionally, muscle aches, chills, sore throat, runny nose, headache, and chest pain were observed [10]. Currently, COVID-19 symptoms are similar to pneumonia, though a significant number of COVID-19 infected cases are asymptomatic, which can affect others as silent spread [11]. Importantly, patients with underlying diseases, such as asthma, diabetes, and hypertension, are more susceptible to COVID-19 [12,13].

A report suggests that around $40 \%$ of those infected with SARS-CoV- 2 might remain asymptomatic and thus, the transmission might carry a greater risk than expected [11]. At present, endeavors to develop medications for COVID-19 by repositioning existing drugs have been pursued, as well as vaccine development. However, the efforts have been hampered by the lack of scientific evidence on how COVID-19 attacks human hosts [14]. On the other hand, phytochemicals have always played a pivotal role in providing potential therapy against diverse diseases [15], including fighting a mutant coronavirus [16]. From folk remedy times, medicinal plants provided many outstanding bioactive compounds, which led to great drug discoveries, such as aspirin from Salix, taxol from Taxus brevifolia, and artemisinin from Artemisia annua [17-19]. During the 2003 SARS outbreak, a series of Chinese herbal treatments were effectively implemented to control the pandemic situation [20], and even now, the Chinese government impulses traditional remedies aimed at COVID-19 prevention. According to the state administration of traditional Chinese medicine (TMC), $85.2 \%$ of the country's COVID-19 patients (total: 60,107$)$ until 17 February 2020 had been recovered with the treatment of TMC [21]. Although researchers race to develop a vaccine, the rigorous validation of traditional medicine for identifying the widespectrum antiviral active components can also be a viable way to control the COVID 19 disease. Therefore, an attempt to explore novel antiviral compounds with the underlying mechanism of action should not be halted.

Korean traditional medicine literature, "Donguibogam", established about 400 years ago, illustrates that Panax Ginseng C.A. Meyer (PGCAM) is a very potent herbal plant with multiple pharmacological activities, particularly strengthening the immune system against some pathogens [22]. According to an experiment, ginseng interrupted cellular oxidative damage induced by a respiratory syncytial virus (RSV) and downregulated pro-inflammatory gene expression level induced by RSV in the human alveolar epithelial cell [23]. Additionally, potential bioactive compounds of ginseng extracts, including ginsenoside derivatives ( $\mathrm{Rg} 1, \mathrm{Rg} 3, \mathrm{Rb} 1, \mathrm{Rb} 2, \mathrm{Rb} 3, \mathrm{Re}, \mathrm{Rd}, \mathrm{Rh} 2$ ) and compound $\mathrm{K}$, are renowned for therapeutic efficacy against several infectious diseases [24]. However, the major drawback of these compounds is their high molecular weight and low bioavailability; even the absorption and transportation rate of intact ginsenosides and their metabolites from the intestines are very poor [25]. In addition, some reports studied via Caco-2 permeability assay indicated the low permeability of ginsenoside derivatives $\left(<2.59 \pm 0.17 \cdot 10^{-7} \mathrm{~cm} / \mathrm{s}\right)$ and compound $\mathrm{K}\left(<8.65 \cdot 10^{-7} \mathrm{~cm} / \mathrm{s}\right)[26,27]$. It is evident that low permeable compounds have poor bioavailability profiles, which do not reach the intended biological system; thus, therapeutic efficacy is not well consistent [28]. Therefore, these findings suggest that the pharmacological value of low molecular weight compounds (LMWCs) in ginseng should be strengthened to provide therapeutic evidence against immune dysfunction, especially in treating COVID-19 infection during an urgent pandemic outbreak.

Network pharmacology (NP), a new paradigm and combined analytical system, can efficiently explore the interaction networking of diverse factors, such as ligands, protein targets, diseases, and genes [29]. NP can unveil the target prediction and mechanism of drug action with a multidimensional perspective, which highlights shifting of the 
approach from "one target, one ligand" to "multiple targets, multivariate therapeutics" [30]. Importantly, it widely contributes to drug discovery by providing $40 \%$ of the recent active candidates with successful clinical evidence [31]. Currently, network pharmacology has been utilized broadly to decipher bioactive compounds and mechanisms of phytochemicals against diverse diseases [32]. In this research, we implemented network pharmacology to analyze LMWCs and mechanisms of PGCAM against COVID-19. Firstly, LMWCs in PGCAM were identified using browsing literature and public database and then filtered through Lipinski's rule for drug-likeness compounds. Afterwards, genes that interacted with selected compounds or COVID-19-related genes were screened using public databases, and the overlapping genes between compounds and COVID-19 target genes were identified. Thirdly, KEGG (Kyoto Encyclopedia of Genes and Genomes) pathway enrichment analysis of overlapping genes was employed to explore the molecular mechanisms of PGCAM against COVID-19. Finally, the potential bioactive compounds and key genes of PGCAM against COVID-19 were selected by analyzing the binding affinity energy in virtual mode. The overall workflow is presented in Figure 1.

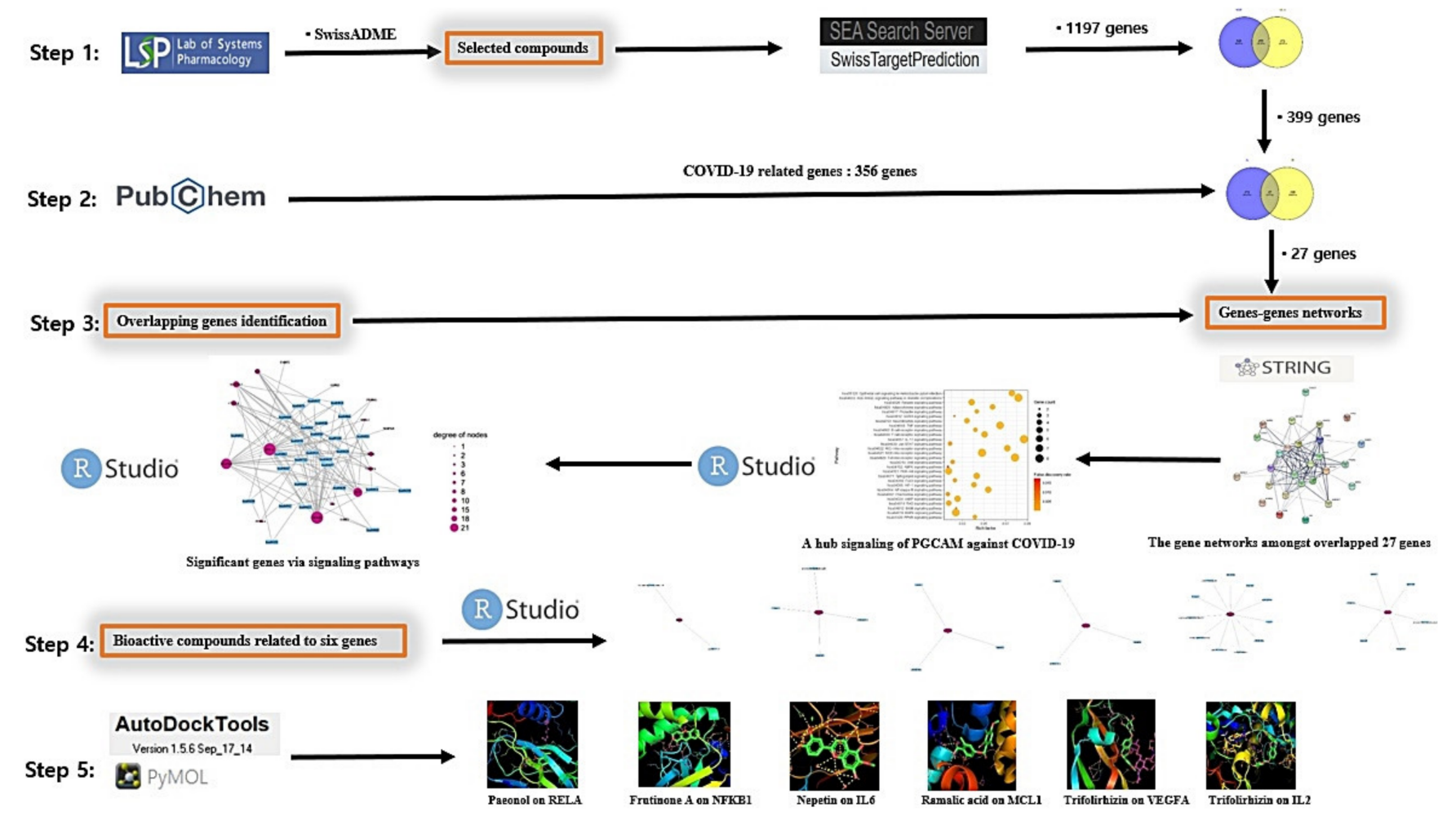

Figure 1. Workflow of network pharmacology analysis of PGCAM against COVID-19.

\section{Results}

\subsection{Potential Low Molecular Weight Bioactive Compounds from PGCAM}

The public databases identified a total of 121 compounds in PGCAM, and the druglikeness properties of 107 compounds were selected by Lipinski's rule, which is presented in Table 1.

\subsection{Target Genes Associated with the 107 Compounds or COVID-19}

As shown in Supplementary Table S1, a total number of 1197 genes (STP + SEA) related to 107 compounds was identified and, among them, the overlapped 399 genes between SEA and STP were screened (Figure 2). The 399 genes (Supplementary Table S2) were again analyzed with COVID-19-related genes (356 genes) (Supplementary Table S3). Finally, the Venn diagram (Figure 3) showed the 27 overlapping genes (Supplementary Table S4) between the 399 genes and the 356 genes. Overall, the final 27 genes were selected from 107 LMWCs of PGCAM through COVID-19-related genes. 
Table 1. A final list of the selected 107 compounds in PGCAM for network analysis based on Lipinski's rule.

\begin{tabular}{|c|c|c|c|c|c|c|c|}
\hline No. & Compound & $\begin{array}{c}\text { Molecular } \\
\text { Weight }(\mathrm{g} / \mathrm{mol})\end{array}$ & Pubchem ID & No. & Compound & $\begin{array}{c}\text { Molecular } \\
\text { Weight (g/mol) }\end{array}$ & Pubchem ID \\
\hline 1 & pisol & 186.33 & 8193 & 55 & protopanaxadiol & 460.7 & 9920281 \\
\hline 2 & $3691-11-0$ & 204.35 & 94275 & 56 & 3,5-dimethyl-p-anisic acid & 180.2 & 88944 \\
\hline 3 & $\varepsilon$-cadinene & 204.35 & 12302130 & 57 & 3-methylheptane & 114.23 & 11519 \\
\hline 4 & $\delta$-elemene & 204.35 & 12309449 & 58 & 4-methyldodecane & 184.36 & 521958 \\
\hline 5 & $(+)$-maalioxide & 222.37 & 6452862 & 59 & 5-(E)-heptadec-12-enyl benzene-1,3-diol & 346.5 & 5318012 \\
\hline 6 & (20S)-protopanaxatriol & 476.7 & 11468733 & 60 & 7-tetradecyne & 194.36 & 141979 \\
\hline 7 & 3,4-dimethylheptane & 128.25 & 13534 & 61 & nepetin & 316.26 & 5317284 \\
\hline 8 & 3-ethyl-3-methylheptane & 142.28 & 140213 & 62 & ZINC8680004 & 456.7 & 7048528 \\
\hline 9 & acetal & 118.17 & 7765 & 63 & $3^{\prime}$-biotin & 244.31 & 5315463 \\
\hline 10 & MAV & 194.14 & 446145 & 64 & celabenzine & 379.5 & 442847 \\
\hline 11 & $\gamma$ - selinene & 204.35 & 521334 & 65 & $(\mathrm{R})-(+)$-citronellal & 154.25 & 75427 \\
\hline 12 & ginsenoyne A & 258.35 & 5317632 & 66 & dianthramine & 289.24 & 441562 \\
\hline 13 & ginsenoyne B & 294.8 & 5317633 & 67 & ditertbutyl phthalate & 278.34 & 121712 \\
\hline 14 & ginsenoyne $\mathrm{C}$ & 276.4 & 5317634 & 68 & arachidonate & 304.5 & 444899 \\
\hline 15 & ginsenoyne D & 262.4 & 5317635 & 69 & frutinone A & 264.23 & 441965 \\
\hline 16 & panaxytriol & 278.4 & 93484 & 70 & folienetriol & 460.7 & 11048822 \\
\hline 17 & methylselenocysteine & 182.09 & 147004 & 71 & (20S)-protopanaxadiol & 460.7 & 11213350 \\
\hline 18 & vulgarin & 264.32 & 94253 & 72 & ginsenoyne $\mathrm{E}$ & 258.35 & 5320336 \\
\hline 19 & palmitic acid & 256.42 & 985 & 73 & girinimbin & 263.3 & 96943 \\
\hline 20 & linoleic acid & 280.4 & 5280450 & 74 & L-erythro-isocitric acid & 192.12 & 439238 \\
\hline 21 & tetradecane & 198.39 & 12389 & 75 & D-erythro-Isocitric acid & 192.12 & 447805 \\
\hline 22 & $\alpha$-gurjunene & 204.35 & 15560276 & 76 & $\begin{array}{l}\text { malvic acid } \\
\text { matu }\end{array}$ & 280.4 & 10416 \\
\hline 23 & $\alpha$-cadinol & 222.37 & 10398656 & 77 & beta-D-mannose & 180.16 & 439680 \\
\hline 24 & loxanol V & 214.39 & 8209 & 78 & methyl tricosanoate & 368.6 & 75519 \\
\hline 25 & 9-hexadecenoic acid & 254.41 & 4668 & 79 & NN-dimethyldecanamide & 199.33 & 26690 \\
\hline 26 & methyl myristate & 242.4 & 31284 & 80 & $\begin{array}{l}\text { stearyl acetate } \\
\text { stut }\end{array}$ & 312.5 & 69968 \\
\hline 27 & pentadecyclic acid & 242.4 & 13849 & 81 & panaxadiol & 460.7 & 73498 \\
\hline 28 & methyl linoleate & 294.5 & 5284421 & 82 & panaxatriol & 476.7 & 73599 \\
\hline 29 & 2,6-dimethyl-3,7-octadiene-2,6-diol & 170.25 & 5352451 & 83 & pancratistatin & 325.27 & 441597 \\
\hline 30 & hydron-propanedioate & 104.06 & 23511544 & 84 & 2 -formylpyrrole & 95.1 & 13854 \\
\hline 31 & methyl stearate & 298.5 & 8201 & 85 & ramalic acid & 346.3 & 5320886 \\
\hline 32 & methyl palmitelaidate & 268.4 & 638303 & 86 & suchilactone & 368.4 & 132350840 \\
\hline 33 & methyl pentadecanoate & 256.42 & 23518 & 87 & trifolirhizin & 446.4 & 442827 \\
\hline 34 & panaxynol & 244.37 & 5281149 & 88 & pangamic acid & 476.6 & 57346693 \\
\hline 35 & bicyclogermacrene & 204.35 & 13894537 & 89 & D-pantothenic Acid & 219.23 & 6613 \\
\hline 36 & neocnidilide & 194.27 & 3083857 & 90 & $\beta$-elemene & 204.35 & 6918391 \\
\hline 37 & OCT & 114.23 & 356 & 91 & $\beta$-santalol & 220.35 & 6857681 \\
\hline 38 & L-adenosine & 267.24 & 448374 & 92 & dammarane & 414.7 & 9548714 \\
\hline 39 & elemicin & 208.25 & 10248 & 93 & methyl margarate & 284.5 & 15609 \\
\hline 40 & diop & 390.6 & 33934 & 94 & $\begin{array}{l}\text { oleanane } \\
\text { ona }\end{array}$ & 412.7 & 9548717 \\
\hline 41 & $\beta$-humulene & 204.35 & 5318102 & 95 & p-glucosyloxymandelonitrile & 311.29 & 441465 \\
\hline 42 & stigmasterol & 412.7 & 5280794 & 96 & darutoside & 484.6 & 44715524 \\
\hline 43 & $\beta$-sitosterol & 414.7 & 222284 & 97 & dibutyl phthalate & 278.34 & 3026 \\
\hline 44 & $\beta$-caryophyllene & 204.35 & 5281515 & 98 & fumarine & 353.4 & 4970 \\
\hline 45 & inermin & 284.26 & 91510 & 99 & paeonol & 166.17 & 11092 \\
\hline 46 & methyl (Z)-icos-11-enoate & 324.5 & 5463047 & 100 & methyl palmitate & 270.5 & 8181 \\
\hline 47 & kaempferol & 286.24 & 5280863 & 101 & N9-formylharman & 212.25 & 129650345 \\
\hline 48 & $\alpha$-humulene epoxide & 220.35 & 14038843 & 102 & adenosine & 267.24 & 60961 \\
\hline 49 & TDA & 214.34 & 12530 & 103 & maltol (3-hydroxy-2-methyl-4-pyrone) & 126.11 & 8369 \\
\hline 50 & n-heptadecanol & 256.5 & 15076 & 104 & salicylic acid & 138.12 & 338 \\
\hline 51 & 1-hexadecyne & 222.41 & 12396 & 105 & p-hydroxycinnamic acid & 164.16 & 637542 \\
\hline 52 & 13-tetradecenyl acetate & 254.41 & 521718 & 106 & gomisin N & 400.5 & 158103 \\
\hline 53 & 2,6,10,15-tetramethylheptadecane & 296.6 & 41209 & 107 & gomisin A & 416.5 & 3001662 \\
\hline 54 & 2-methyltridecane & 198.39 & 15269 & & & & \\
\hline
\end{tabular}

\subsection{Potential Molecular Pathways of PGCAM against COVID-19}

The gene-gene networking analysis through overlapping 27 genes was visualized by STRING, which includes 24 nodes and 122 edges (Figure 4). The 3 (PTPRS, CPA3, and S1PR3) out of 27 genes were not associated with each other. The KEGG pathway enrichment analysis via STRING suggested that 27 overlapping genes were significantly enriched in 28 signaling pathways (Figure 5). Detailed descriptions of the 28 signaling pathways are listed in Table 2. Among them, both RELA and NFKB1 connected directly to 21 signaling pathways out of 28 signaling pathways and were noted as hub genes of PGCAM against the COVID-19 (Figure 6). Coincidently, both RELA and NFKB1 play vital roles in all 21 signaling pathways by the PI3K-Akt signaling pathway, indicating that the PI3K-Akt signaling pathway might be a hub signaling pathway of PGCAM against COVID-19. 


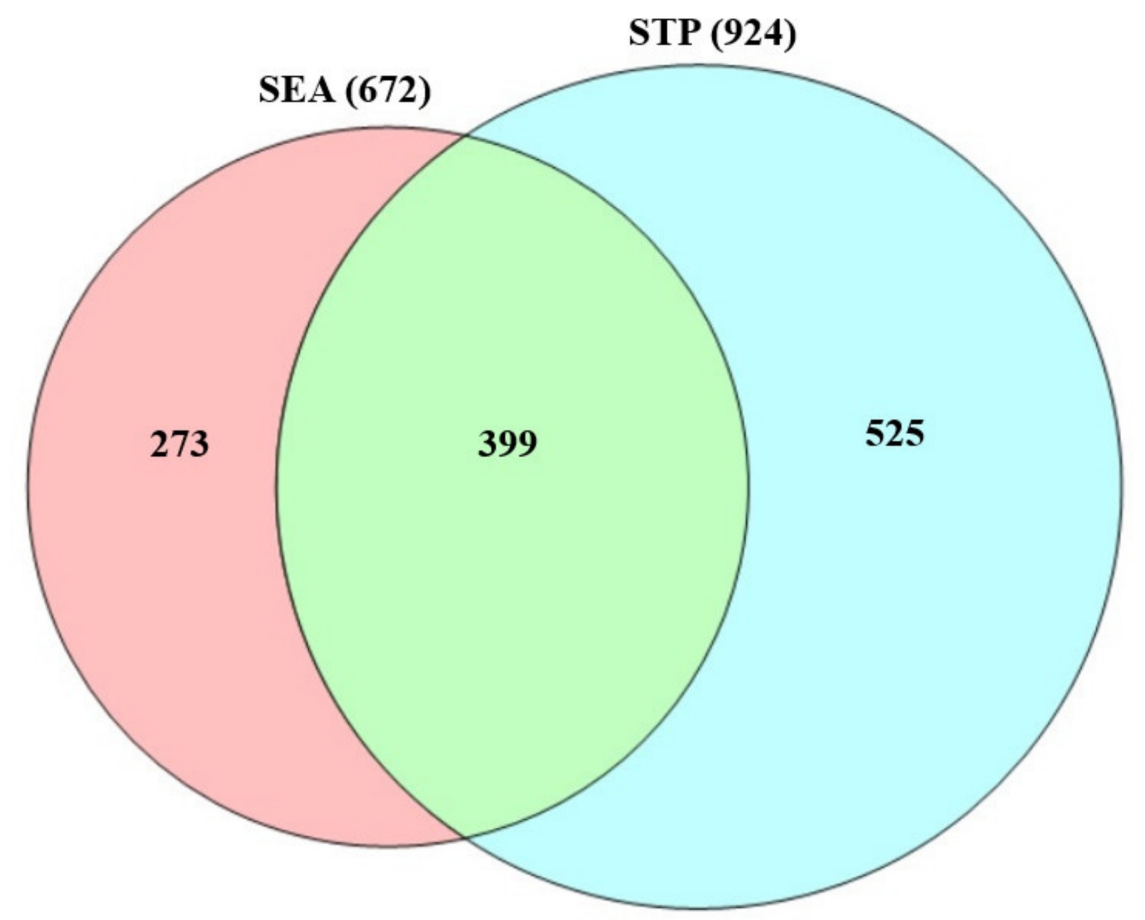

Figure 2. Overlapping genes (399 genes) between SEA (672 genes) and STP (924 genes).

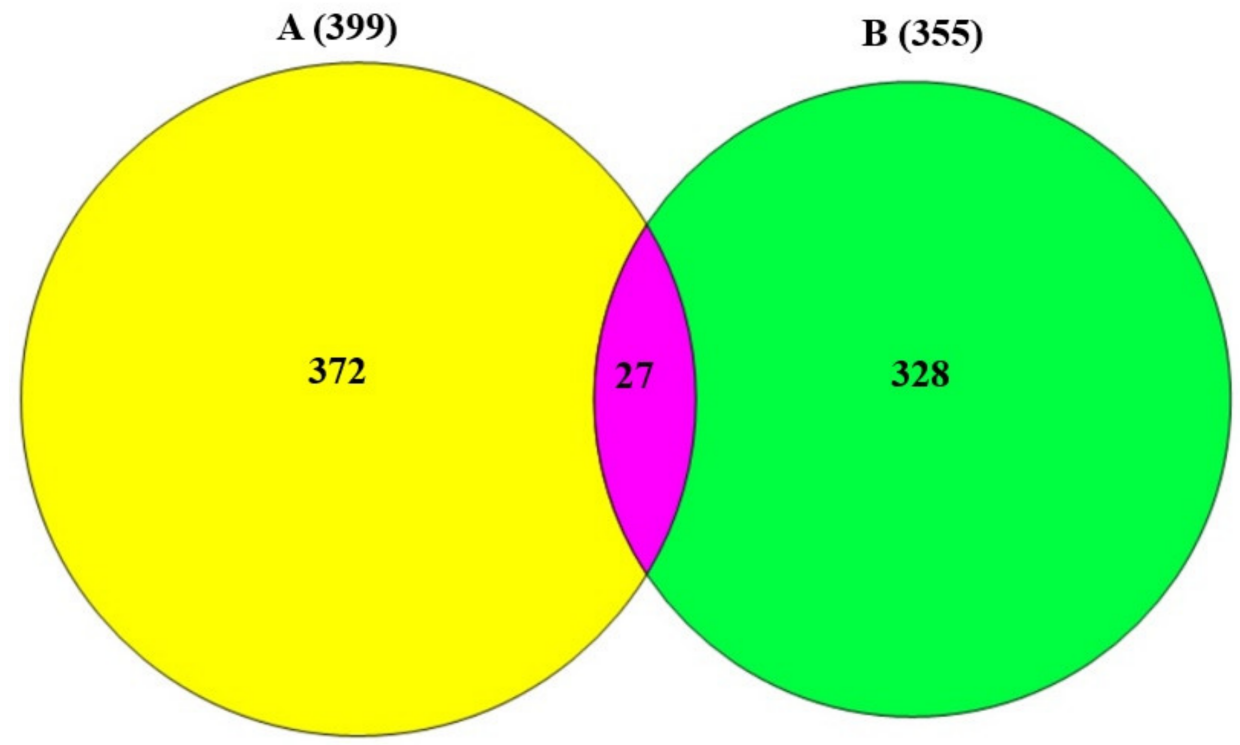

Figure 3. Overlapping genes between 399 overlapping genes from two databases (A) and COVID-19related genes (356 genes) (B). * Both human and mouse ACE2 gene included in Venn diagram, thus indicating a total of 355 genes $(\mathbf{B})$. 


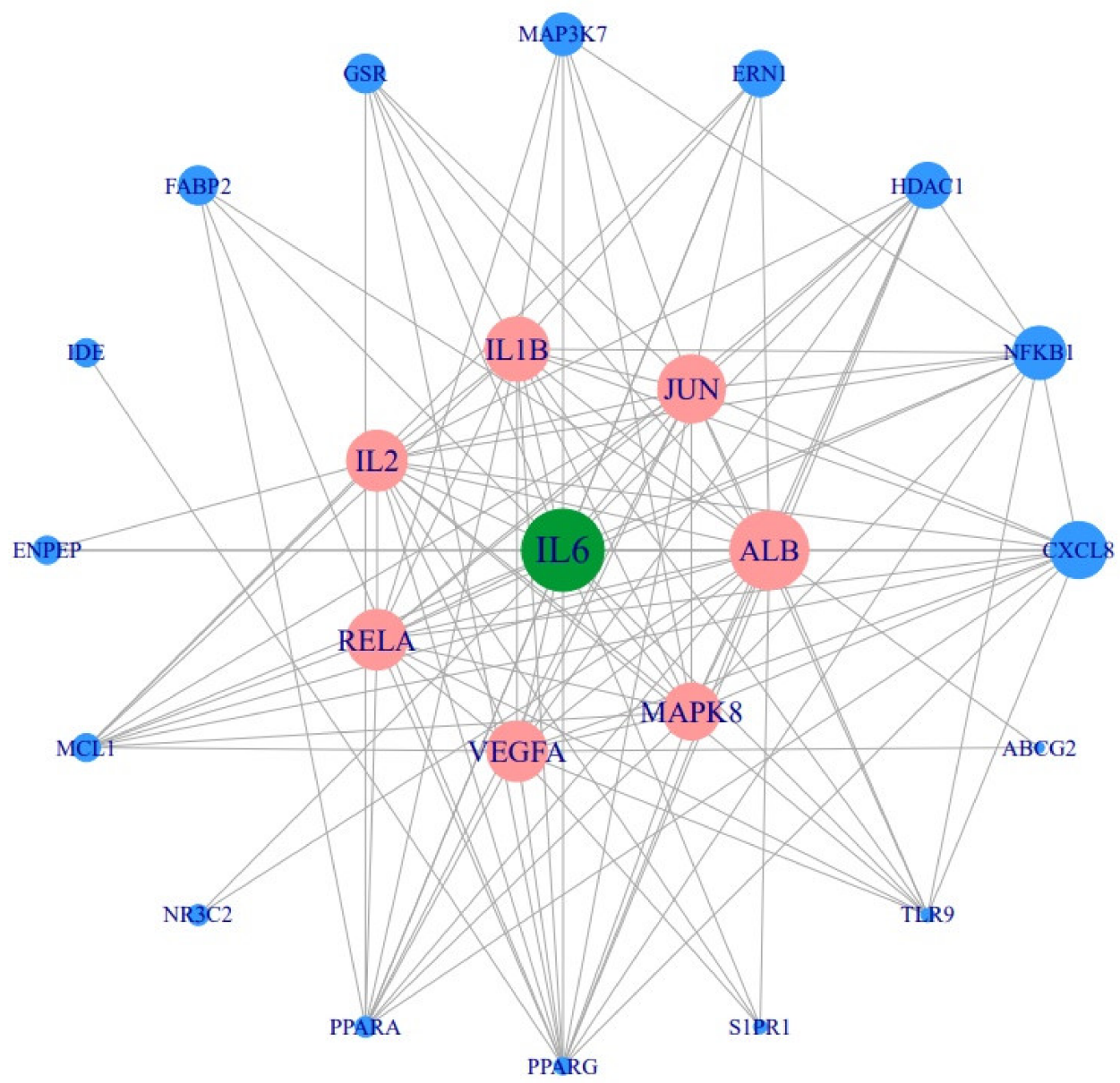

Figure 4. Gene-gene interaction with 27 nodes and 117 edges in PGCAM against COVID-19.

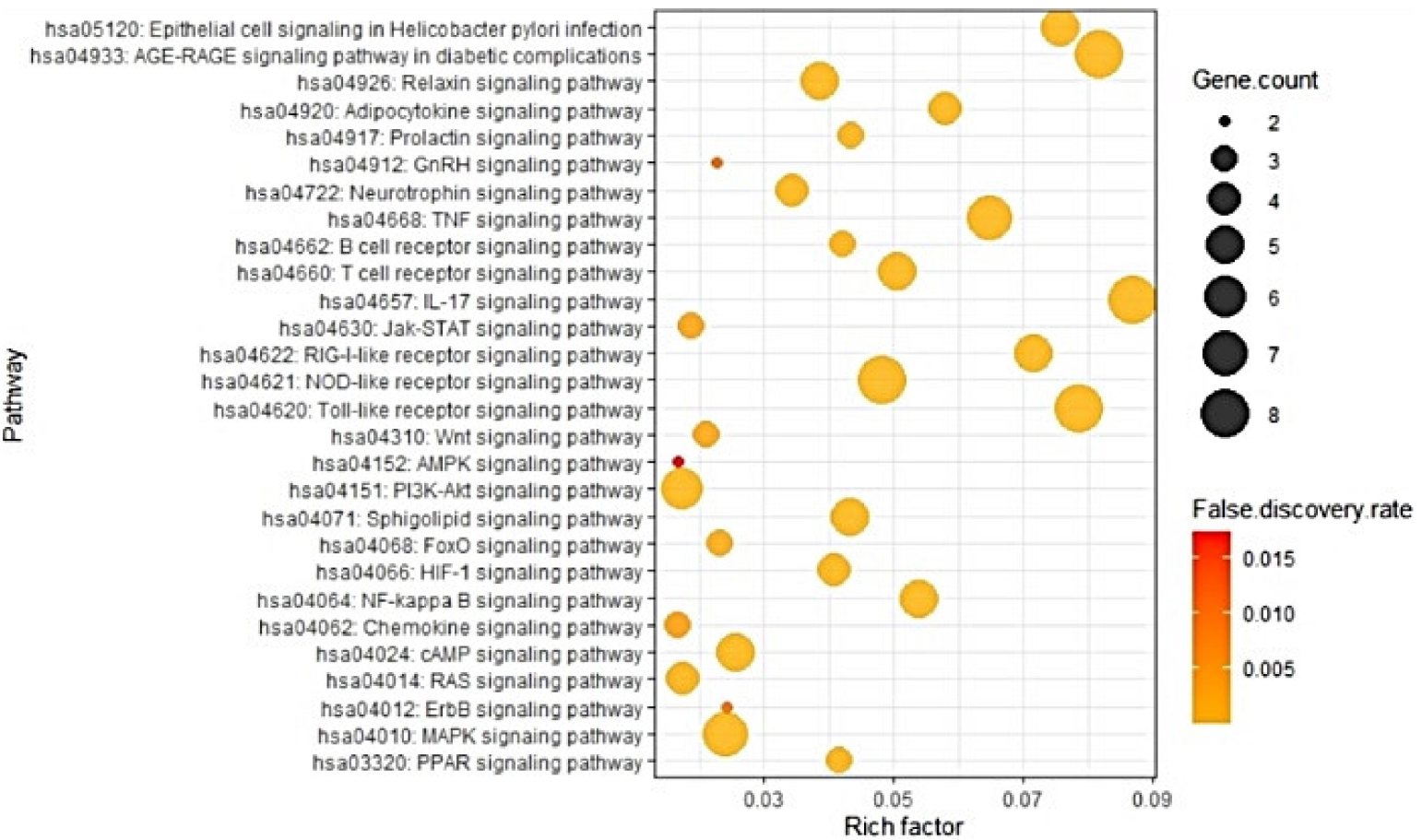

Figure 5. Bubble chart of 28 signaling pathways in PGCAM against COVID-19. 
Table 2. Target genes in 28 signaling pathways enrichment related to COVID-19.

\begin{tabular}{|c|c|c|}
\hline KEGG ID & Description & Target Genes \\
\hline hsa04933 & AGE-RAGE signaling pathway in diabetic complications & RELA, MAPK8, NFKB1, IL1B, JUN, VEGFA, CXCL8 \\
\hline hsa04657 & IL-17 signaling pathway & RELA, MAPK8, NFKB1, IL1B, JUN, IL6, CXCL8, MAP3K7 \\
\hline hsa04620 & Toll-like receptor signaling pathway & RELA, MAPK8, NFKB1, IL1B, JUN, IL6, CXCL8, MAP3K7 \\
\hline hsa04621 & NOD-like receptor signaling pathway & RELA, MAPK8, NFKB1, IL1B, JUN, IL6, CXCL8, MAP3K7 \\
\hline hsa04668 & TNF signaling pathway & RELA, MAPK8, NFKB1, IL1B, JUN, IL6, MAP3K7 \\
\hline hsa05120 & Epithelial cell signaling in Helicobacter pylori infection & RELA, JUN, MAPK8, NFKB1, CXCL8 \\
\hline hsa04622 & RIG-I-like receptor signaling pathway & RELA, NFKB1, MAPK8, MAP3K7, CXCL8 \\
\hline hsa04010 & MAPK signaling pathway & RELA, MAPK8, NFKB1, IL1B, JUN, VEGFA, MAP3K7 \\
\hline hsa04064 & NF-kappa B signaling pathway & RELA, NFKB1, IL1B, CXCL8, MAP3K7 \\
\hline hsa04660 & $\mathrm{T}$ cell receptor signaling pathway & RELA, NFKB1, JUN, IL2,MAP3K7 \\
\hline hsa04071 & Sphigolipid signaling pathway & RELA, NFKB1,MAPK8,S1PR1,S1PR3 \\
\hline hsa04926 & Relaxin signaling pathway & RELA, NFKB1,JUN, MAPK8, VEGFA \\
\hline hsa04920 & Adipocytokine signaling pathway & RELA, NFKB1, MAPK8, PPARA \\
\hline hsa04151 & PI3K-Akt signaling pathway & RELA, NFKB1, IL6, MCL1, VEGFA, IL2 \\
\hline hsa04024 & cAMP signaling pathway & RELA, NFKB1, JUN, MAPK8, PPARA \\
\hline hsa04066 & HIF-1 signaling pathway & RELA, NFKB1, IL6, VEGFA \\
\hline hsa04722 & Neurotrophin signaling pathway & RELA, NFKB1,JUN, MAPK8 \\
\hline hsa04917 & Prolactin signaling pathway & RELA, NFKB1, MAPK8 \\
\hline hsa04662 & B cell receptor signaling pathway & RELA, NFKB1,JUN \\
\hline hsa03320 & PPAR signaling pathway & PPARA, PPARG, FABP2 \\
\hline hsa04014 & RAS signaling pathway & RELA, NFKB1, MAPK8, VEGFA \\
\hline hsa04068 & FoxO signaling pathway & IL6, MAPK8, Ś1PR1 \\
\hline hsa04310 & Wnt signaling pathway & JUN, MAPK8, MAP3K7 \\
\hline hsa04630 & Jak-STÅT signaling pathway & IL2, IL6, MCL1 \\
\hline hsa04062 & Chemokine signaling pathway & RELA, NFKB1, CXCL8 \\
\hline hsa04012 & ErbB signaling pathway & JUN, MAPK8 \\
\hline hsa04912 & GnRH signaling pathway & JUN, MAPK8 \\
\hline hsa04152 & AMPK signaling pathway & PPARG, MAP3K7 \\
\hline
\end{tabular}

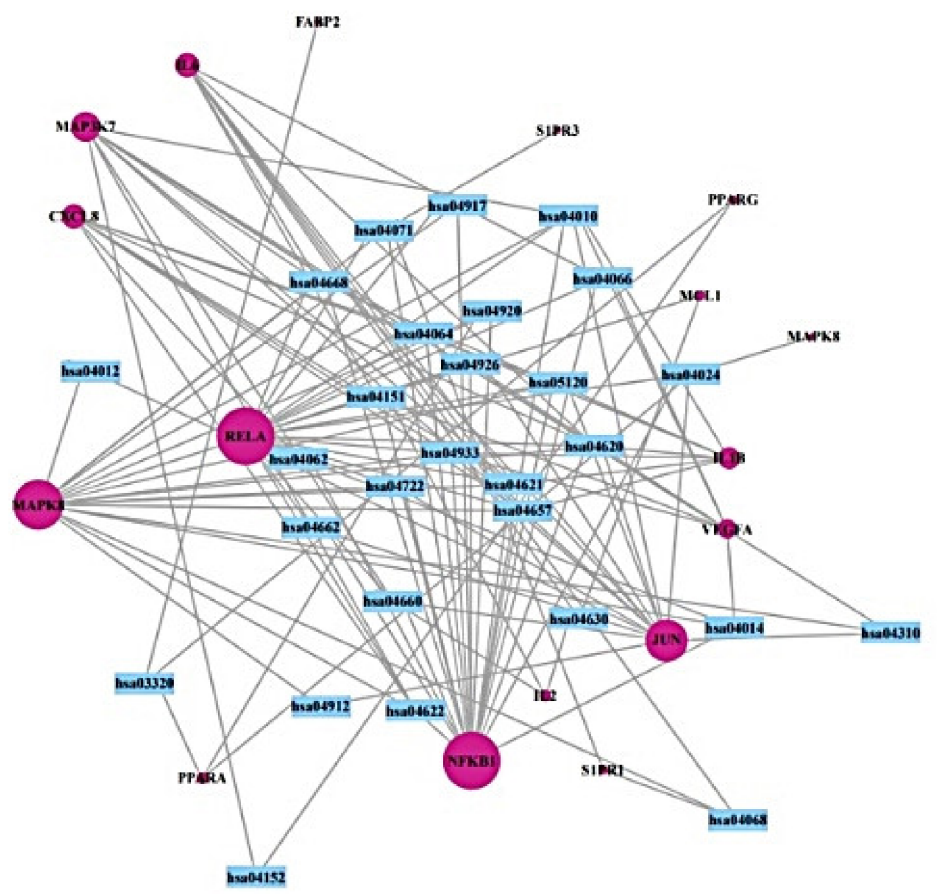

Degree of values

. 1

- 2

- 3

- 6

- 7

- 8

- 10

- 15

- 18

21

Figure 6. Degree values of 17 genes in 28 signaling pathways.

\subsection{Key Genes and Bioactive Compounds of PGCAM against COVID-19}

Based on the PI3K-Akt signaling pathway, six key genes (RELA, NFKB1, IL6, MCL1, VEGFA, and IL2) and 24 potential key bioactive compounds were identified in PGCAM against COVID-19. The 24 compounds are associated with six key genes on PI3K-Akt signaling pathway. The 24 compounds (Figure 7) are classified as follows: eight aliphatic acyclic compounds (NN-dimethyldecanamide, linoleic acid, methyl palmitelaidate, methyl linoleate, methyl myristate, methyl stearate, methyl margarate, and methyl pentadecanoate), eight aromatic heteropolycyclic compounds (frutinone A, nepetin, L-adenosine, adenosine, N9-formylharman, inermin, trifolirhizin, and kaempferol), five aromatic ho- 
momonocyclic compounds (paeonol, p-hydroxycinnamic acid, dibuthyl phthalate, salicylic acid, and ramalic acid), one aliphatic heteromonocyclic compound (beta-D-mannose), one aliphatic heteropolycyclic compound (darutoside), and one aromatic heteromonocyclic compound (p-glucosyloxymandelonitrile). The interactions between each gene and potential active compound were visualized with RStudio (Supplementary Figure S1).

(A)

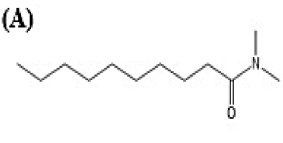

NN-dimethyldecanamide
(B)

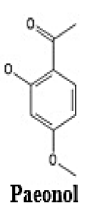

(C)

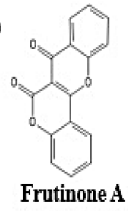

(J)

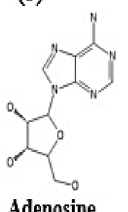

Adenosine

(N)

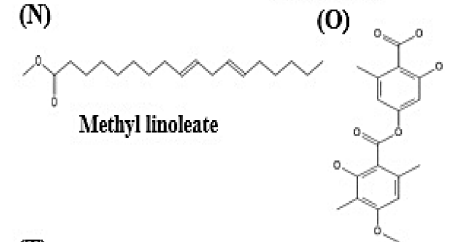

(T)

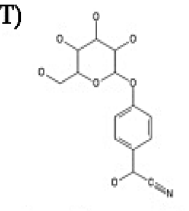

p-glucosyloxymandelonitril

(X)<smiles>[CH]1CCC1</smiles>

Methyl margarate

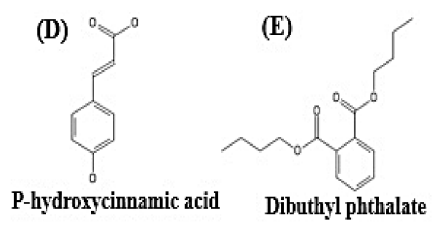

(K)

(L)

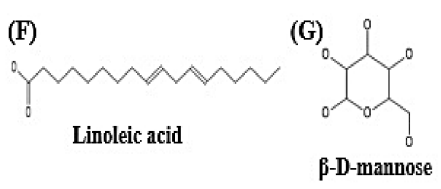

(M)

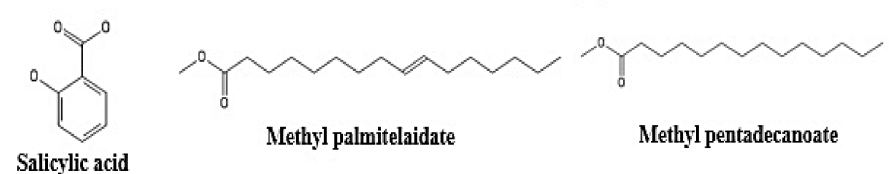

(Q)
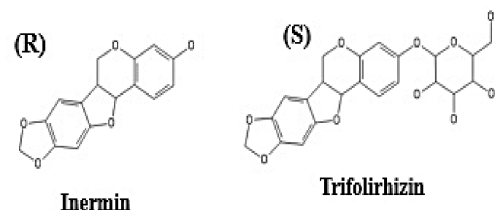

(W)

(V)

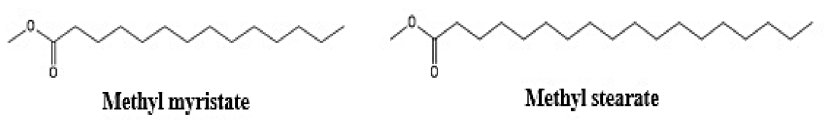

Figure 7. Chemical structures of potential bioactive compounds in PGCAM against COVID-19.

2.5. Affinity Score on Six Key Genes of Potential Bioactive Compounds in PGCAM against COVID-19

The potential bioactive compounds were docked against six genes (RELA, NFKB1, IL6, MCL1, VEGFA, IL2) to measure the binding energy. The molecular docking figure is presented in Figure 8. 

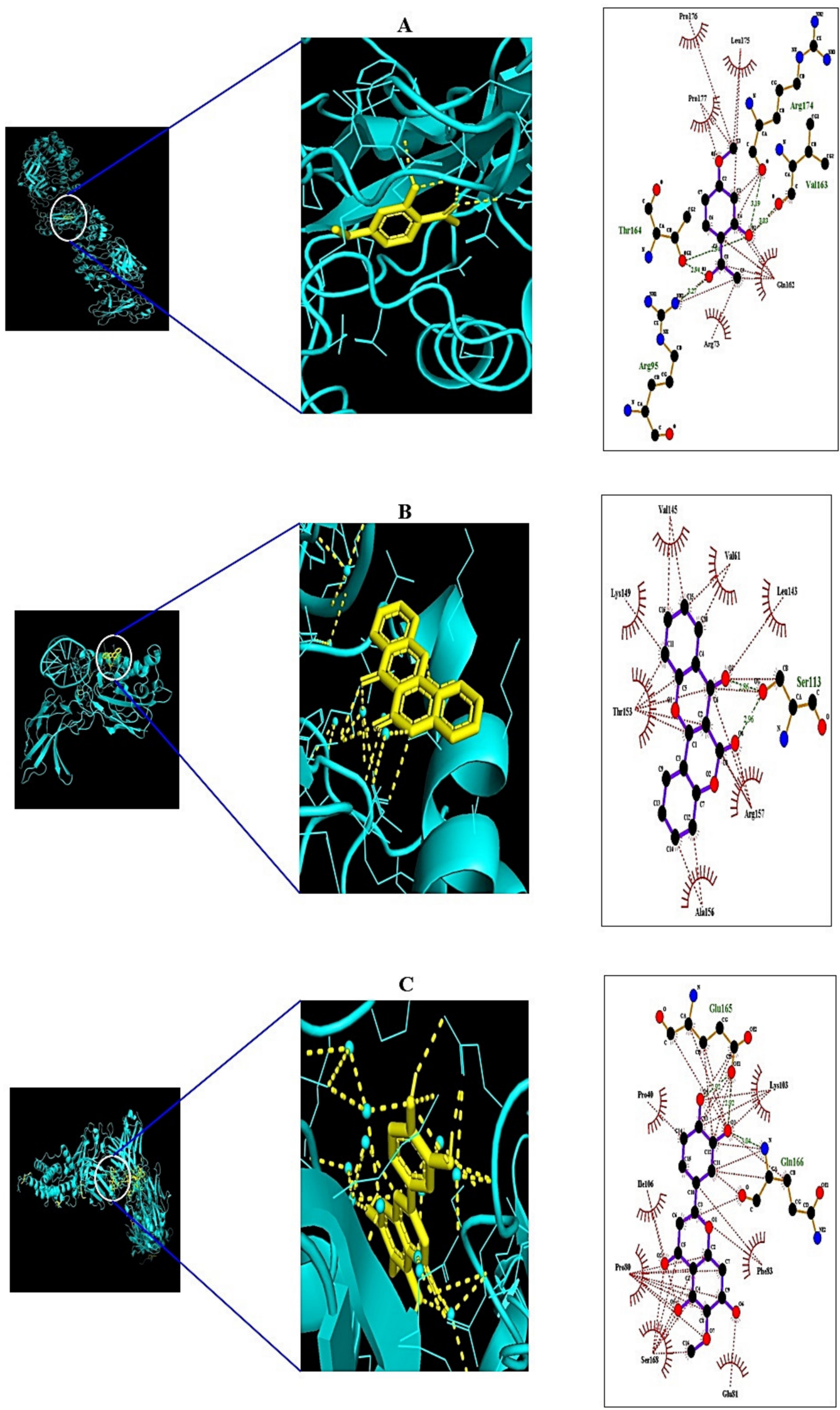

Figure 8. Cont. 

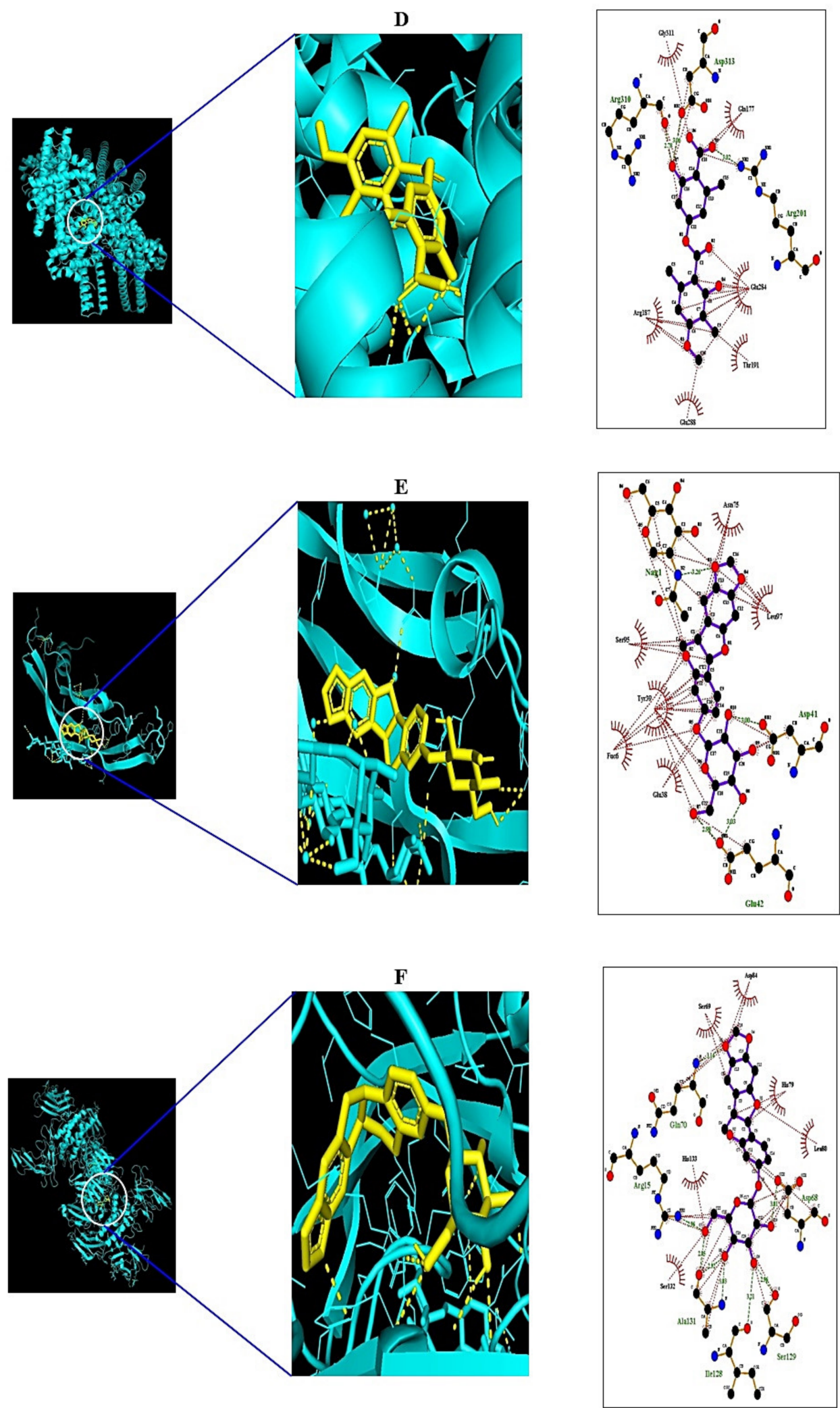

Figure 8. Molecular docking interaction between best docked compounds from PGCAM and target proteins. (A) Paeonol on 1NFI (B) Frutinone A on 1SVC (C) Nepetin on 4CNI (D) Ramalic acid on 5JSB (E) Trifolirhizin on 4KZN (F) Trifolirhizin on 2ERJ.

Docking simulation of the affinity between R1-R2 and RELA protein in the "Homo sapiens" setting was analyzed. Based on the docking score, the order of priority of binding 
energy is given as: R2, R1. The two-binding energy of R1-RELA and R2-RELA demonstrated -3.9 and $-5.3 \mathrm{kcal} / \mathrm{mol}$, respectively. Interaction analysis of the best-docked compound, namely "Paeonol", resulted in four hydrogen bonds (Arg-174, Val-163, Thr-164, Arg-95) and seven hydrophobic bonds (Pro-176, Leu-175, Pro-177, Gln-162, Arg-73). The detailed information is listed in Table 3. Docking simulation of the affinity between N1-N4 and NFKB1 protein in the "Homo sapiens" setting revealed promising binding affinity, and the order of priority of binding energy is as follows: N1, N3, N2, N4. The binding energy of N1-NFKB1, N2-NFKB1, N3-NFKB1, and N4-NFKB1 exposed $-7.2,-5.2,-5.6$, and $-4.6 \mathrm{kcal} / \mathrm{mol}$, respectively. Interaction analysis of the best-docked compound, namely "Frutinone A", resulted in one hydrogen bond (Ser-113) and seven hydrophobic bonds (Val-145, Val-61, Leu-143, Arg-157, Ala-156, Thr-153, Lys-149). The detailed information is listed in Table 4. Docking simulations of the affinity between L1-L3 and IL6 protein in the "Homo sapiens" setting showed good binding energy, and the order of priority is given as: L3, L2, L1. The five-binding energy of L1-IL6, L2-IL6, and L3-IL6 were - 5.0, - 5.7, and $-8.0 \mathrm{kcal} / \mathrm{mol}$, respectively. Interaction analysis of the best-docked compound, namely "Nepetin", resulted in two hydrogen bonds (Glu-165, Gln-166) and seven hydrophobic bonds (Lys-103, Phe-83, Glu-81, Ser-168, Pro-80, Ile-106, Pro-40). The detailed information is listed in Table 5. Docking simulation of the affinity between M1-M8 and MCL1 protein in the "Homo sapiens" setting was investigated, and the order of priority of binding energy is as follows: M7, M1, M2 = M8, M3, M6, M5, M4. The eight-binding energy of M1-MCL1, M2MCL1, M3-MCL1, M4-MCL1, M5-MCL1, M6-MCL1, M7-MCL1, and M8-MCL1 unveiled $-6.8,-6.5,-5.3,-4.2,-4.4,-4.7,-6.9$, and $-6.5 \mathrm{kcal} / \mathrm{mol}$, respectively. Interaction analysis of the best-docked compound, namely "Ramalic acid", resulted in three hydrogen bonds (Asp-313, Arg-201, Arg-310) and five hydrophobic bonds (Gly-311, Glu-284, Thr-191, Glu-288, Arg-187). The detailed information is listed in Table 6.

Table 3. Binding energy of potential active compounds on RELA (PDB ID:1NFI).

\begin{tabular}{|c|c|c|c|c|c|c|}
\hline & & & & & $\begin{array}{l}\text { Hydrogen Bond } \\
\text { Interactions }\end{array}$ & $\begin{array}{l}\text { Hydrophobic } \\
\text { Interactions }\end{array}$ \\
\hline Protein & Ligand & PubChem ID & Symbol & $\begin{array}{c}\text { Binding Energy } \\
(\mathrm{kcal} / \mathrm{mol})\end{array}$ & $\begin{array}{c}\text { Amino Acid } \\
\text { Residue }\end{array}$ & Amino Acid Residue \\
\hline \multirow[t]{3}{*}{ 1NFI } & $\begin{array}{c}\text { NN- } \\
\text { dimethyldecanamide }\end{array}$ & 26690 & R1 & -3.9 & Thr-67 & Gly-68, Pro-69 \\
\hline & & & & & & $\begin{array}{c}\text { Lys-314, Arg-166 } \\
\text { Pro-317, Tyr-66 } \\
\text { Asp-167 }\end{array}$ \\
\hline & Paeonol & 11092 & $\mathrm{R} 2$ & -5.3 & $\begin{array}{c}\text { Arg-174, Val-163 } \\
\text { Thr-164, Arg-95 }\end{array}$ & $\begin{array}{l}\text { Pro-176, Leu-175 } \\
\text { Pro-177, Gln-162 } \\
\text { Arg-73 }\end{array}$ \\
\hline
\end{tabular}

Docking simulation of the affinity between V1-V11 and VEGFA protein in the "Homo sapiens" setting was explored, and the order of priority of binding energy is as follows: V4, V3, V2 = V6 = V7, V5, V9, V1, V11, V8 = V10. The eleven-binding energy of V1VEGFA, V2-VEGFA, V3-VEGFA, V4-VEGFA, V5-VEGFA, V6-VEGFA, V7-VEGFA, V8VEGFA, V9-VEGFA, V10-VEGFA, and V11-VEGFA revealed -5.0, -7.1, -7.5, -8.1, -6.8, $-7.1,-7.1,-3.9,-5.1,-3.9$, and $-4.4 \mathrm{kcal} / \mathrm{mol}$, respectively. Interaction analysis of the best-docked compound, namely "Trifolirhizin", resulted in two hydrogen bonds (Asp-41, Glu-42) and five hydrophobic bonds (Asn-75, Glu-38, Tyr-39, Ser-95, Leu-97). The detailed information is listed in Table 7. 
Table 4. Binding energy of potential active compounds on NFKB1 (PDB ID: 1SVC).

\begin{tabular}{|c|c|c|c|c|c|c|}
\hline & & & & & $\begin{array}{c}\text { Hydrogen Bond } \\
\text { Interactions }\end{array}$ & $\begin{array}{l}\text { Hydrophobic } \\
\text { Interactions }\end{array}$ \\
\hline Protein & Ligand & PubChem ID & Symbol & $\begin{array}{c}\text { Binding Energy } \\
(\mathrm{kcal} / \mathrm{mol})\end{array}$ & $\begin{array}{c}\text { Amino Acid } \\
\text { Residue }\end{array}$ & $\begin{array}{c}\text { Amino Acid } \\
\text { Residue }\end{array}$ \\
\hline \multirow[t]{4}{*}{$1 S V C$} & Frutinone A & 441965 & N1 & -7.2 & Ser-113 & $\begin{array}{c}\text { Val-145, Val-61 } \\
\text { Leu-143, Arg-157 } \\
\text { Ala-156, Thr-153 } \\
\text { Lys-149 }\end{array}$ \\
\hline & Paeonol & 11092 & N2 & -5.2 & $\begin{array}{c}\text { Ser-113, Arg-157 } \\
\text { Thr-153 }\end{array}$ & $\begin{array}{c}\text { His-144, Lys-149 } \\
\text { Ala-111, His-112 } \\
\text { Leu-143, Val-145 } \\
\text { Val-61 }\end{array}$ \\
\hline & $\begin{array}{l}\mathrm{P}- \\
\text { hydroxycinnamic } \\
\text { acid }\end{array}$ & 637542 & N3 & -5.6 & $\begin{array}{c}\text { Ala-111, Ser-113 } \\
\text { Arg-157 }\end{array}$ & $\begin{array}{c}\text { His-144, Lys-149 } \\
\text { Leu-143, Thr-146 } \\
\text { Thr-153, His-112 } \\
\text { Val-145, Val-61 }\end{array}$ \\
\hline & $\begin{array}{l}\text { Dibuthyl } \\
\text { phthalate }\end{array}$ & 3026 & N4 & -4.6 & Lys-337 & $\begin{array}{c}\text { Lys-278, Phe-301 } \\
\text { Ile-281, Pro-303 } \\
\text { Gly-299, Phe-298 } \\
\text { Gln-282 }\end{array}$ \\
\hline
\end{tabular}

Table 5. Binding energy of potential active compounds on IL6 (PDB ID: 4CNI).

\begin{tabular}{|c|c|c|c|c|c|c|}
\hline & & & & & $\begin{array}{l}\text { Hydrogen Bond } \\
\text { Interactions }\end{array}$ & Hydrophobic Interactions \\
\hline Protein & Ligand & PubChem ID & Symbol & $\begin{array}{c}\text { Binding Energy } \\
\text { (kcal/mol) }\end{array}$ & $\begin{array}{c}\text { Amino Acid } \\
\text { Residue }\end{array}$ & Amino Acid Residue \\
\hline \multirow[t]{3}{*}{$4 \mathrm{CNI}$} & Linoleic acid & 5280450 & L1 & -5.0 & Lys-39 & $\begin{array}{c}\text { Glu-81, Pro-80 } \\
\text { Ser-168, Phe-83 } \\
\text { Gln-105, Leu-104 } \\
\text { Gln-166, Lys-103 } \\
\text { Glu-165, Pro-40 }\end{array}$ \\
\hline & $\beta$-d-mannose & 439680 & L2 & -5.7 & $\begin{array}{c}\text { Val-170, Asp-167 } \\
\text { Asn-138, Asp-170 } \\
\text { Ser-168, Gly-169 }\end{array}$ & His-171, Lys-169 \\
\hline & Nepetin & 5317284 & L3 & -8.0 & Glu-165, Gln-166 & $\begin{array}{c}\text { Lys-103, Phe-83 } \\
\text { Glu-81, Ser-168 } \\
\text { Pro-80, Ile-106 } \\
\text { Pro-40 }\end{array}$ \\
\hline
\end{tabular}

Table 6. Binding energy of potential active compounds on MCL1 (PDB ID: 5JSB).

\begin{tabular}{|c|c|c|c|c|c|c|}
\hline & & & & & $\begin{array}{c}\text { Hydrogen Bond } \\
\text { Interactions }\end{array}$ & $\begin{array}{l}\text { Hydrophobic } \\
\text { Interactions }\end{array}$ \\
\hline Protein & Ligand & PubChem ID & Symbol & $\begin{array}{l}\text { Binding Energy } \\
\text { (kcal/mol) }\end{array}$ & $\underset{\text { Residue }}{\text { Amino Acid }}$ & $\begin{array}{c}\text { Amino Acid } \\
\text { Residue }\end{array}$ \\
\hline \multirow[t]{4}{*}{ 5JSB } & L-adenosine & 448374 & M1 & -6.8 & $\begin{array}{c}\text { Gly-192, Ser-202 } \\
\text { Tyr-116, Gln-189 } \\
\text { Glu-188 }\end{array}$ & $\begin{array}{l}\text { Arg-201, Glu-115 } \\
\text { Arg-222, Gln-221 } \\
\text { Lys-276, Asp-218 }\end{array}$ \\
\hline & Adenosine & 60961 & M2 & -6.5 & $\begin{array}{l}\text { Ser-202, Gly-192 } \\
\text { Glu-188, Tyr-116 } \\
\text { Asp-218, Gln-189 }\end{array}$ & Lys-276, Arg-222 \\
\hline & Salicylic acid & 338 & M3 & -5.3 & Ala-193, Thr-191 & $\begin{array}{c}\text { Glu-173, Asp-195 } \\
\text { Arg-184, Arg-187 } \\
\text { Glu-188 }\end{array}$ \\
\hline & Methyl palmitelaidate & 638303 & M4 & -4.2 & Lys-102 & $\begin{array}{c}\text { Tyr-106, Arg-101 } \\
\text { Ala-99, Glu-98 } \\
\text { Lys-11, Ala-14 } \\
\text { Val-18, Leu-95 } \\
\text { Glu-15 }\end{array}$ \\
\hline
\end{tabular}


Table 6. Cont.

\begin{tabular}{|c|c|c|c|c|c|c|}
\hline & & & & & $\begin{array}{c}\text { Hydrogen Bond } \\
\text { Interactions }\end{array}$ & $\begin{array}{l}\text { Hydrophobic } \\
\text { Interactions }\end{array}$ \\
\hline \multirow[t]{6}{*}{ Protein } & Ligand & PubChem ID & Symbol & $\begin{array}{c}\text { Binding Energy } \\
(\mathrm{kcal} / \mathrm{mol})\end{array}$ & $\begin{array}{c}\text { Amino Acid } \\
\text { Residue }\end{array}$ & $\begin{array}{c}\text { Amino Acid } \\
\text { Residue }\end{array}$ \\
\hline & $\begin{array}{c}\text { Methyl } \\
\text { pentadecanoate }\end{array}$ & 23518 & M5 & -4.4 & Tyr-106 & Val-18, Ala-99 \\
\hline & & & & & & $\begin{array}{c}\text { Glu-98, Arg-101 } \\
\text { Lys-11, Glu-15 } \\
\text { Ala-14, Leu-95 } \\
\text { Lys-102 }\end{array}$ \\
\hline & Methyl linoleate & 5284421 & M6 & -4.7 & Lys-102, Tyr-106 & $\begin{array}{c}\text { Glu-15, Ala-99 } \\
\text { Leu-95, Glu-98 } \\
\text { Lys-11, Ala-14 } \\
\text { Arg-101, Val-18 }\end{array}$ \\
\hline & Ramalic acid & 5320886 & M7 & -6.9 & $\begin{array}{c}\text { Asp-313, Arg-201 } \\
\text { Arg-310 }\end{array}$ & $\begin{array}{c}\text { Gly-311, Glu-284 } \\
\text { Thr-191, Glu-288 } \\
\text { Arg-187 }\end{array}$ \\
\hline & N9-formylharman & 129650345 & M8 & -6.5 & $\mathrm{n} / \mathrm{a}$ & $\begin{array}{l}\text { Arg-187, Asp-195 } \\
\text { Glu-188, Arg-184 } \\
\text { Ala-193, Glu-173 } \\
\text { Arg-201, Thr-191 }\end{array}$ \\
\hline
\end{tabular}

Table 7. Binding energy of potential active compounds on VEGFA (PDB ID: 4KZN).

\begin{tabular}{|c|c|c|c|c|c|c|}
\hline & & & & & $\begin{array}{l}\text { Hydrogen Bond } \\
\text { Interactions }\end{array}$ & $\begin{array}{l}\text { Hydrophobic } \\
\text { Interactions }\end{array}$ \\
\hline Protein & Ligand & PubChem ID & Symbol & $\begin{array}{l}\text { Binding Energy } \\
\text { (kcal/mol) }\end{array}$ & $\begin{array}{l}\text { Amino Acid } \\
\text { Residue }\end{array}$ & $\begin{array}{c}\text { Amino Acid } \\
\text { Residue }\end{array}$ \\
\hline \multirow[t]{11}{*}{$4 \mathrm{KZN}$} & $\beta$-d-mannose & 439680 & V1 & -5.0 & $\begin{array}{l}\text { Asn-75, Ser-95 } \\
\text { Tyr-39, Glu-38 }\end{array}$ & Phe-96, Leu-97 \\
\hline & Darutoside & 44715524 & $\mathrm{~V} 2$ & -7.1 & Asp-41, Ser-95 & $\begin{array}{c}\text { Glu-42, Ile-43 } \\
\text { Glu-38, Tyr-39 } \\
\text { Phe-96 }\end{array}$ \\
\hline & Inermin & 91510 & V3 & -7.5 & $\begin{array}{l}\text { Glu-38, Leu-97 } \\
\text { Asp-41 }\end{array}$ & $\begin{array}{l}\text { Phe-96, Ser-95 } \\
\text { Pro-40, Tyr-39 }\end{array}$ \\
\hline & Trifolirhizin & 442827 & V4 & -8.1 & Asp-41, Glu-42 & $\begin{array}{c}\text { Asn-75, Glu-38 } \\
\text { Tyr-39, Ser-95 } \\
\text { Leu-97 }\end{array}$ \\
\hline & P-glucosyloxymandelonitrile & 441465 & V5 & -6.8 & $\begin{array}{l}\text { Leu-97, Asn-75 } \\
\text { Asp-41, Glu-38 }\end{array}$ & $\begin{array}{c}\text { Pro-40, Glu-42 } \\
\text { Ser-95, Phe-96 } \\
\text { Tyr-39 }\end{array}$ \\
\hline & Nepetin & 5317284 & V6 & -7.1 & $\mathrm{n} / \mathrm{a}$ & $\begin{array}{l}\text { Asp-41, Tyr-39 } \\
\text { Phe-96, Leu-97 }\end{array}$ \\
\hline & Kaempferol & 5280863 & V7 & -7.1 & Asn-75 & $\begin{array}{l}\text { Phe-96, Ser-95 } \\
\text { Tyr-39, Asp-41 }\end{array}$ \\
\hline & Methyl myristate & 31284 & V8 & -3.9 & $\mathrm{n} / \mathrm{a}$ & $\begin{array}{c}\text { Asn-75, Glu-38 } \\
\text { Tyr-39, Ser-95 } \\
\text { Leu-97 }\end{array}$ \\
\hline & Methyl stearate & 8201 & V9 & -5.1 & $\mathrm{n} / \mathrm{a}$ & $\begin{array}{c}\text { Glu-42, Asp-41 } \\
\text { Glu-38, Asn-75 } \\
\text { Leu-97, Ser-95 } \\
\text { Tyr-39 }\end{array}$ \\
\hline & Methyl pentadecanoate & 23518 & V10 & -3.9 & $\mathrm{n} / \mathrm{a}$ & $\begin{array}{c}\text { Tyr-39, Glu-38 } \\
\text { Leu-97, Ser-95 } \\
\text { Asn-75 }\end{array}$ \\
\hline & Methyl margarate & 15609 & V11 & -4.4 & $\mathrm{n} / \mathrm{a}$ & $\begin{array}{l}\text { Glu-73, Tyr-39 } \\
\text { Asn-75, Leu-97 }\end{array}$ \\
\hline
\end{tabular}

Finally, docking simulation of the affinity between P1-P6 and IL2 protein in the "Homo sapiens" setting was investigated, and the order of priority of binding energy is as follows: P2, P5, P1 = P4, P3, P6. The six-binding energy of P1-IL2, P2-IL2, P3-IL2, P4-IL2, P5-IL2, and P6-IL2 indicated $-7.0,-8.5,-6.3,-7.0,-7.4$, and $-5.2 \mathrm{kcal} / \mathrm{mol}$, respectively. Interaction analysis of best-docked compound, namely "Trifolirhizin", resulted in six hydrogen bonds 
(Asp-68, Ser-129, Ile-128, Ala-131, Arg-15, Gln-70) and six hydrophobic bonds (Ser-69, Asp-84, His-79, Leu-80, Ser-132, His-133). The detailed information is listed in Table 8.

Table 8. Binding energy of potential active compounds on IL2 (PDB ID: 2ERJ).

\begin{tabular}{|c|c|c|c|c|c|c|}
\hline & & & & & $\begin{array}{l}\text { Hydrogen Bond } \\
\text { Interactions }\end{array}$ & $\begin{array}{l}\text { Hydrophobic } \\
\text { Interactions }\end{array}$ \\
\hline Protein & Ligand & PubChem ID & Symbol & $\begin{array}{l}\text { Binding Energy } \\
(\mathrm{kcal} / \mathrm{mol})\end{array}$ & $\begin{array}{l}\text { Amino Acid } \\
\text { Residue }\end{array}$ & $\begin{array}{l}\text { Amino Acid } \\
\text { Residue }\end{array}$ \\
\hline \multirow[t]{6}{*}{ 2ERJ } & Darutoside & 44715524 & P1 & -7.0 & $\begin{array}{c}\text { Ile-63, Gln-46 } \\
\text { Val-88, Arg-85 } \\
\text { Trp-90,Thr-47 } \\
\text { Asn-61 }\end{array}$ & Gln-34, Cys-48 \\
\hline & Trifolirhizin & 442827 & $\mathrm{P} 2$ & -8.5 & $\begin{array}{c}\text { Asp-68, Ser-129 } \\
\text { Ile-128, Ala-131 } \\
\text { Arg-15, Gln-70 }\end{array}$ & $\begin{array}{c}\text { Ser-69, Asp-84 } \\
\text { His-79, Leu-80 } \\
\text { Ser-132, His-133 }\end{array}$ \\
\hline & \multicolumn{2}{|c|}{$\begin{array}{c}\text { P- } \\
\text { glucosyloxymandelonitrile } \\
441465\end{array}$} & P3 & -6.3 & $\begin{array}{l}\text { Asn-61, Arg-85 } \\
\text { Trp-90, Glu-49 }\end{array}$ & $\begin{array}{l}\text { Cys-48, Ile-63 } \\
\text { Gln-34 }\end{array}$ \\
\hline & Nepetin & 5317284 & $\mathrm{P} 4$ & -7.0 & Pro-109, Gln-199 & $\begin{array}{c}\text { Ile-110, Leu-112 } \\
\text { Met-107, Pro-196 } \\
\text { Trp-197, Ala-108 } \\
\text { Ser-198 }\end{array}$ \\
\hline & Kaempferol & 5280863 & P5 & -7.4 & $\begin{array}{l}\text { Asp-20, Arg-15 } \\
\text { His-133 }\end{array}$ & $\begin{array}{c}\text { Leu-85, Asp-84 } \\
\text { Ser-69, Gln-70 } \\
\text { Arg-81, His-79 } \\
\text { Ala-66, Asp-68 } \\
\text { Met-23 }\end{array}$ \\
\hline & Methyl linoleate & 5284421 & P6 & -3.7 & $\mathrm{n} / \mathrm{a}$ & $\begin{array}{c}\text { Cys-60, Cys-48 } \\
\text { Leu-51, Glu-49 } \\
\text { Ile-63, Asn-61 }\end{array}$ \\
\hline
\end{tabular}

This result illustrated that the five bioactive compounds might be anti-inflammatory agents against COVID-19 (Figure 9).

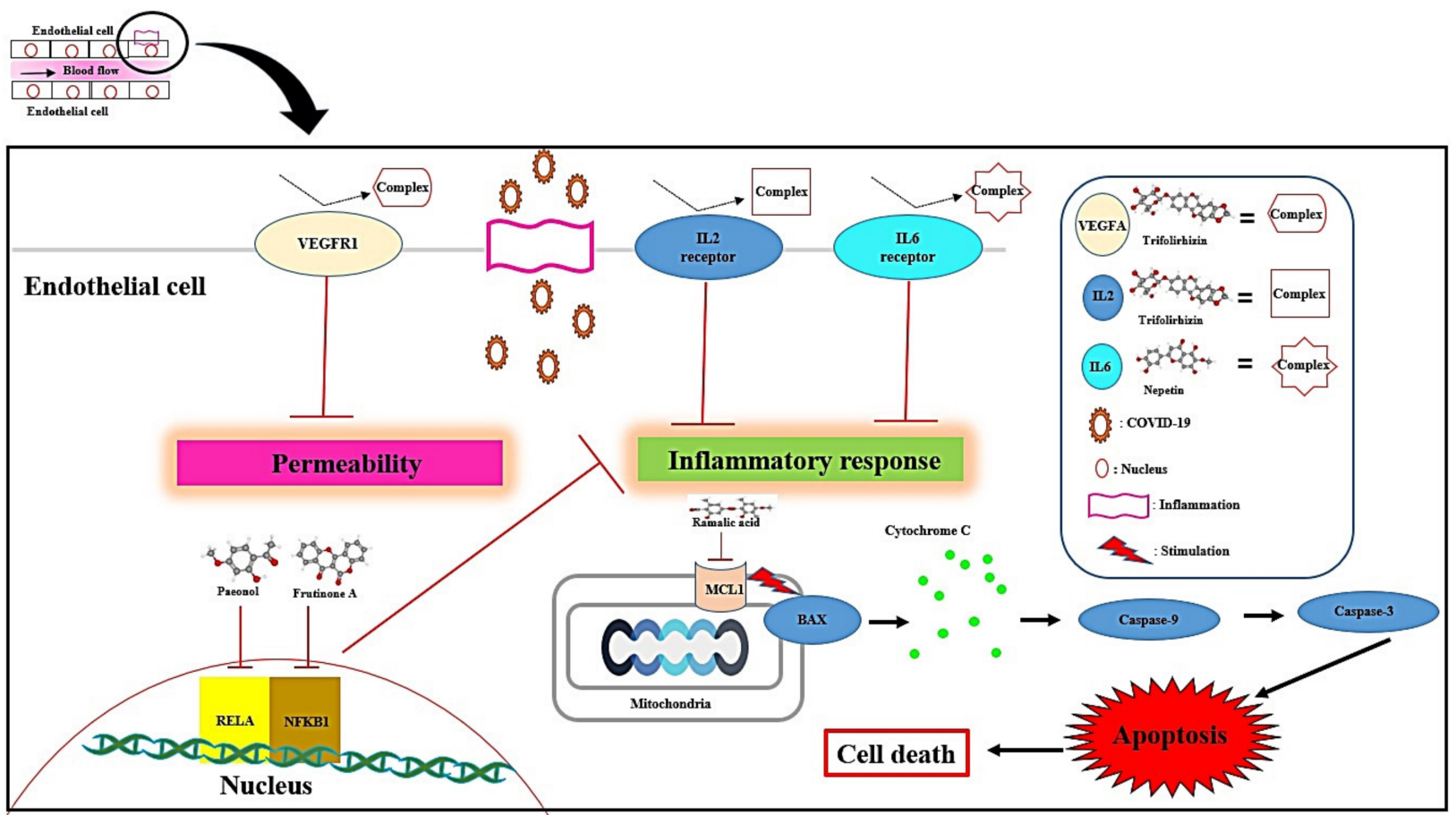

Figure 9. Illustration of key findings in this study.

\section{Discussion}

Compounds-genes network indicated that the clinical efficacy of PGCAM on COVID19 -associated genes was directly involved in 27 genes. Of these, both RELA and NFKB1 
genes were more significant genes than any other kind of genes. On top of that, based on the pathway enrichment, the PI3K-Akt signaling pathway is found as the topmost mechanism of PGCAM against COVID-19. Accordingly, the docking score through virtual screening on the six genes related to the PI3K-Akt signaling pathway suggested that five bioactive compounds (paeonol, frutinone A, nepetin, ramalic acid, trifolirhizin) were considered the most significant compounds of PGCAM against COVID-19. Meanwhile, the consequence of the KEGG pathway enrichment analysis of 27 genes might be an anti-COVID mechanism. The relationships of 28 signaling pathways with anti-virus properties were discussed as follows.

PPAR signaling pathway: Triggered PPAR- $\alpha$ regulates immunity of cytoplasmic DNA, blocks interferon production, and boosts vulnerability to viral infection [33].

MAPK (Mitogen-Activated Protein Kinase) signaling pathway: Virus-infected cells cause severe reactions that activate the MAPK signaling cascades to shield from virus attack. They can also be used by viruses to facilitate viral replication [34]. Furthermore, the p38 MAPK signaling pathway is a potent inflammatory pathway in lung and heart damage in COVID-19 patients [35].

ErbB signaling pathway: A great number of prime oncogenic virus infections are associated with ErbB signaling pathway. The Hepatitis B Virus(HBV) also induces overexpression of the Epidermal Growth Factor Receptor (EGFR) gene and protein [34,36-38].

RAS signaling pathway: Inhibition of RAS can curtail tissue damage severity in COVID-19 patients. Additionally, ACE (Angiotensin-converting enzyme) blockers cause a reduction in the response of the RAS system [39].

cAMP signaling pathway: cAMPs might have a viral-resistant effect by curbing viral replication and interrupting viral entry. On the other hand, they might have an inimical role by decreasing HIV antiviral immune responses, thus halting the removal of the virus and causing $\mathrm{T}$ cell malfunction [40].

Chemokine signaling pathway: Elevation of chemokines (CXCL10 and CXCL8) is clinically characteristic of COVID-19 infection. Moreover, its expression level indicated different clinical acuteness among the COVID-19 patients [41].

NF- $\kappa B$ signaling pathway: Activation of NF- $\kappa B$ signaling pathway shows acute inflammation induced after SARS-CoV attack, and NF- $\kappa B$ blockers are favorable antivirals in the disorder caused by COVID-19 [42].

HIF-1 signaling pathway: HIF-1 $\alpha$ expression supports SARS-CoV-2 replication and sustainability, and it intensifies the cytokine production level in monocytes [43].

FOXO signaling pathway: Reduction of FOXO3 in T cells interrupted apoptosis, elevated multifunction of CD8 cells, and enhanced viral control [44].

Sphingolipid signaling pathway: Sphingolipids play a major protective role in the lungs against pulmonary and lung damage, and the control of their pathways may provide effective and improved therapeutic tactics [45]. Regulation of sphingolipids can be very advantageous and can have an effect on anti-inflammation, preservation of neuronal integrity, and anti-coagulant effects [46,47]. All of these favorable features might be utilized to counterbalance the involved disorders of COVID-19 [48].

PI3K-Akt signaling pathway: Activating of PI3K-Akt signaling is an important mechanism to prolong viral replication in both acute and constant infection, thus, virus-infected Vero E6 cells are activated by PI3K-Akt pathway [49,50].

AMPK signaling pathway: In both HBV and HBC viral infections, the AMPK activation is advantageous, but in others, such as the Ebola virus, dengue virus, and even human cytomegaloviral infections, AMPK plays a counteracting role [51].

Wnt signaling pathway: Controlling Wnt signaling could be a critical process in affecting the sustainability of viral pathogenesis [52].

Toll-like receptor signaling pathway: TLR-7 agonists may inhibit the onset of acute COVID-19 in indicative patients and synergy effects with antiviral treatment [53]. 
NOD-like receptor signaling pathway: NLRs may regulate inflammatory response via NF- $\mathrm{B}$ inhibition and block the viral defense system via interaction with mitochondrial antiviral-signaling protein (MAVS) [54].

RIG-I-like receptor signaling pathway: A report shows that SARS-CoV-2 ORF6 blocks RIG-I-like receptor signaling pathway, thus leading to the interruption of the host's innate immune system [55].

JAK/STAT signaling pathway: JAK/STAT pathway is linked to the induction of multiple molecular immune pathways. The interruption of this pathway may lead to the blockade of several cellular responses [56].

IL-17 signaling pathway: IL-17 blockers are immunologically probable as a strategy to inhibit severe respiratory symptoms in COVID-19 patients [57].

$\mathrm{T}$ cell receptor signaling pathway: It remains uncertain whether $\mathrm{T}$ cell responses are beneficial or detrimental in COVID-19, and whether T cell responses have minimal or maximal effects in COVID-19 infection [58].

B cell receptor signaling pathway: B cells have a crucial role in fighting viral infections; however, B cells are triggered after viral infection and before the production of immunoglobulin $\mathrm{G}(\mathrm{IgG})$ [59].

TNF signaling pathway: Anti-TNF $\alpha$ monoclonal antibodies are likely to weaken inflammatory disorders occurring in COVID-19 infection, lessening the release of other inflammatory-aggravating triggers [60].

Neutrophin signaling pathway: A report shows that neurofilament light chain (NfL)a biomarker of astrocytic and neuronal damage-was elevated in the blood of patients with COVID-19 [61]. It is evident that COVID-19 infection might dampen the neurological signaling pathway.

GnRH signaling pathway: When viral infections disturb the Blood-Brain Barrier (BBB), circulating immune cells, such as B and T cells, monocytes, and granulocytes, can enter the brain parenchyma to produce cytokines, and thus, it might be able to negatively alter the functions of GnRH neurons [62].

Prolactin signaling pathway: Patients with HIV have a higher prolactin amount compared to the healthy. Additionally, prolactin is considered as a cytokine in its response in the immune system $[63,64]$.

Adipocytokine signaling pathway: Adipose tissue can react to proinflammatory inducement triggered in the lung through systemic circulation adipocytokines and other inflammatory factors [65].

Relaxin signaling pathway: The dysfunction of the relaxin signaling pathway might bring respiratory disorders recognized in COVID-19 patients. Moreover, SARS-CoV-2 NSP7 protein renders relaxin receptors very much weaker [66].

AGE-RAGE signaling pathway in diabetic complications: AGE-RAGE can also act as an innate immune sensor, including in respiratory viruses. As a consequence, the blocking of RAGE curtails the inflammatory level and progression of cardiovascular diseases [67].

Epithelial cell signaling in Helicobacter pylori infection: Helicobacter pylori downregulates $\mathrm{T}$ and $\mathrm{B}$ cell signaling to initiate the immune system [68]. It is obvious that COVID-19 patients with Helicobacter pylori might be vulnerable to inflammatory responses.

Based on the pathway enrichment analysis, both RELA and NFKB1 were considered as hub genes in PGCAM against COVID-19. Two genes of each were directly enriched in 21 out of 28 signaling pathways by the PI3K-Akt signaling pathway, indicating that PI3K-Akt signaling pathway might be a hub signaling pathway in PGCAM against COVID-19. The other four genes (IL6, MCL1, VEGFA, and IL2) directly related to the PI3K-Akt signaling pathway might be the significant genes to exert synergistic effects against COVID-19. Liu et al. [69] showed that inhibition of both RELA and/or NFKB1 reduces cytokine secretion and thus alleviates inflammation severity. A report specified that MCL-1 inhibition in mitochondrial membrane stimulates BAX, Cytochrome C, Caspase-9, and Caspase-3, and thus it leads to cell death $[70,71]$. Noticeably, a report indicated that vascular endothelial growth factor A (VEGFA) was inhibited by angiotensin-converting enzyme 2 (ACE2) and 
upregulated by the attack of COVID-19, since COVID-19 interrupts the expression of ACE2. Consequently, VEGFA elevates vascular permeability and aggravation of endothelial damage [72]. Most recently, it has been reported that a higher level of both IL-2 and IL-6 was detected in COVID-19 patients [73]. Endothelial cell inflammation is also a major severity in COVID-19 infection, and its unmanageable cytokine production in tissues and cells aggravates severe immune reaction, which is defined as "cytokine storm", resulting in worsening pneumonia. On top of that, the inhibition of six genes (RELA, NFKB1, IL6, MCL1, VEGFA, and IL2) associated with the PI3K-Akt signaling pathway contributes to the anti-proinflammation, anti-vascular permeability, and pro-apoptosis against COVID-19.

Therefore, the key mechanism of PGCAM against COVID-19 might be to block inflammation and vascular permeability, and trigger pro-apoptosis in tissues and/or cells by inactivating the PI3K-Akt signaling pathway.

\section{Materials and Methods}

\subsection{Selective Compound Construction and Drug-Likeness Evaluation}

The LMWCs information of PGCAM was collected by TCMSP (https: / tcmspw.com/ tcmsp.php), literature, SMILES, and Pubchem CID. The molecular formula of selective compounds was identified using ChemSpider (https:/ / www.chemspider.com/StructureSearch. aspx, accessed on 13 October 2021) or Pubchem (https:/ / pubchem.ncbi.nlm.nih.gov, accessed on 14 October 2021). The drug-likeness properties of the identified compounds were selected through Lipinski's rule (molecular weight $\leq 500 \mathrm{~g} / \mathrm{mol}$; Moriguchi octanolwater partition coefficient $\leq 4.15$; the number of nitrogen or oxygen $\leq 10$; and the number of $\mathrm{NH}$ or $\mathrm{OH} \leq 5$ ) in SwissADME (http://www.swissadme.ch, accessed on 14 October 2021). The compound structures were drawn by PubChem Sketcher V2.4 (https:/ / pubchem.ncbi.nlm.nih.gov/edit3/index.html accessed on 14 October 2021).

\subsection{Target Genes Related to Selected Compounds or COVID-19}

Based on SMILES, target genes associated with screened compounds were selected through both SEA (http:/ / sea.bkslab.org, accessed on 15 October 2021) and STP (http: / / www.swisstargetprediction.ch, accessed on 15 October 2021) with "Homo Sapiens" mode. The COVID-19-related genes were identified through PubChem (https:/ / pubchem.ncbi. nlm.nih.gov, accessed on 17 October 2021). The overlapping genes between compounds and COVID-19 target genes were identified and visualized by VENNY 2.1 (https:/ / bioinfogp. cnb.csic.es/tools/venny, accessed on 18 October 2021).

\subsection{Bubble Chart of Enrichment Pathway Analysis of Overlapping Genes}

Genes-genes interaction figure was drawn by STRING (https://string-db.org, accessed on 22 October 2021). RStudio plotted the bubble chart of KEGG (Kyoto Encyclopedia of Genes and Genomes) pathway enrichment analysis of overlapping genes. The results of KEGG pathway enrichment provide a hint at the promising molecular mechanism of PGCAM against COVID-19.

\subsection{Binding Affinity Energy Value of Key Bioactive Compounds on Genes In Silico}

The binding affinity energy measurement of the uttermost potential bioactive compounds on key genes was determined by Autodock (http: / / autodock.scripps.edu, accessed on 25 October 2021), Vina (http: / / vina.scripps.edu/ accessed on 25 October 2021), Pymol (https://pymol.org/2/ accessed on 25 October 2021).

\section{Conclusions}

The efficiency of LMWCs extracted from PGCAM and their anti-COVID-19 mechanisms were firstly analyzed by using network pharmacology. The findings of this research indicated that potential bioactive compounds are paenol, frutinone A, ramalic acid, nepetin, and trifolirhizin, and the target genes are RELA, NFKB1, MCL1, VEGFA, IL2, and IL6 of PGCAM against COVID-19. The mechanism(s) of PGCAM against COVID-19 might 
be inhibited inflammation, vascular permeability, and induction of cell apoptosis against COVID-19 by inactivating the PI3K-Akt signaling pathway. This research provides a scientific indication to support the therapeutic effect of PGCAM on COVID-19, and thus, the proper application of five bioactive compounds against COVID-19 might have potential synergistic effects, such as anti-inflammation, anti-vascular permeability, and pro-apoptosis of the infected cells to prevent COVID-19 vulnerability.

Still, there are also limitations to our analysis. The incompleteness of the herbal natural products database, COVID-19-related genes and protein-protein interaction network might bring bias about target gene findings. Moreover, our approach is dependent on GSEA analysis and molecular docking simulation, either agonist or antagonist. Thus, our predicted result would need to be further improved, through in vitro and in vivo.

The current study did not verify the LMWCs of PGCAM, whose efficacy needs to be validated via clinical test. However, our approach is a holistic perspective and integrated gene-compound interaction, which might be good and promising hints against COVID-19.

Finally, our analysis did not consider the target gene expression level practically after treatment of the selected compounds, which should be implemented in the future.

Supplementary Materials: The following supporting information can be downloaded at: https: / /www.mdpi.com/article/10.3390/pr10020333/s1, Table S1: 1:197 genes (STP + SEA) related to 107 compounds. Table S2: The overlapping genes of SEA and STP: 399 Genes. Table S3: COVID 19-related genes: 356 genes. Table S4: Final 27 overlapping genes related to COVID-19. Figure S1: (A) Interactions between two bioactive compounds and RELA (PDB ID: 1NFI). (B) Interactions between four bioactive compounds and NFKB1 (PDB ID: 1SVC). (C) Interactions between three bioactive compounds and IL6 (PDB ID: 4CNI). (D) Interactions between nine bioactive compounds and MCL1 (PDB ID: 5JSB). (E) Interactions between eleven bioactive compounds and VEGFA (PDB ID: 4KZN). (F) Interactions between six bioactive compounds and IL2 (PDB ID: 2ERJ).

Author Contributions: Conceptualization, methodology, formal analysis, investigation, data curation, writing - original draft, K.-K.O.; software, investigation, data curation, K.-K.O. and M.A.; validation, writing - review and editing, M.A.; supervision, project administration, D.-H.C. All authors have read and agreed to the published version of the manuscript.

Funding: This research did not receive any specific grant from funding agencies in the public, commercial, or not-for-profit sectors.

Institutional Review Board Statement: Not applicable.

Informed Consent Statement: Not applicable.

Data Availability Statement: All data generated or analyzed during this study are included in this published article (and its Supplementary Information files).

Acknowledgments: This study has been worked with the support of a research grant of Kangwon National University in 2022.

Conflicts of Interest: There are no conflicts of interest declared.

$\begin{array}{ll}\text { Abbreviations } \\ \text { ACE2 } & \text { Angiotensin-Converting Enzyme 2 } \\ \text { AGE } & \text { Advanced Glycation Endproducts } \\ \text { AMPK } & \text { AMP-activated Protein Kinase } \\ \text { Caco-2 cell } & \text { Human intestinal epithelial cell } \\ \text { cAMP } & \text { Cyclic Adenosine MonoPhosphate } \\ \text { cAMPs } & \text { Cyclic Adenosine MonoPhosphates } \\ \text { CD8 } & \text { Cluster of Differentiation 8 } \\ \text { COVID-19 } & \text { SARS-CoV-2 virus } \\ \text { EGFR } & \text { Epidermal Growth Factor Receptor } \\ \text { FOXO } & \text { FOrkhead boX protein O } \\ \text { GnRH } & \text { Gonadotropin-Releasing Hormone } \\ \text { ErbB } & \text { Erythroblastic Leukemia Viral Oncogene Homolog }\end{array}$




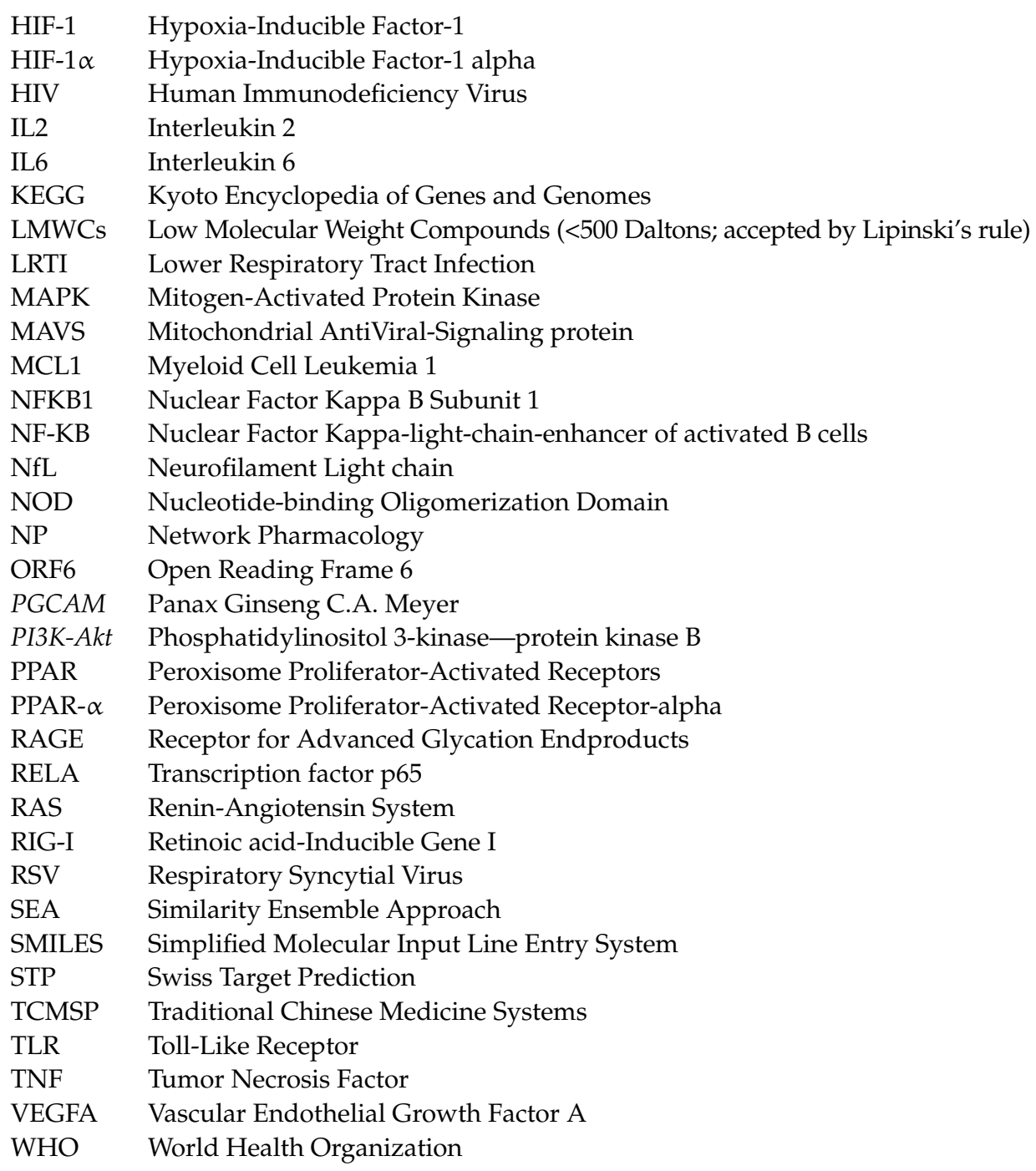

\section{References}

1. Belongia, E.A.; Naleway, A.L. Smallpox Vaccine: The Good, the Bad, and the Ugly. Clin. Med. Res. 2003, 1, 87. [CrossRef]

2. Keni, R.; Alexander, A.; Nayak, P.G.; Mudgal, J.; Nandakumar, K. COVID-19: Emergence, Spread, Possible Treatments, and Global Burden. Front. Public Health 2020, 8, 1-13. [CrossRef] [PubMed]

3. Wu, Y.C.; Chen, C.S.; Chan, Y.J. The outbreak of COVID-19: An overview. J. Chin. Med. Assoc. 2020, 83, 217-220. [CrossRef] [PubMed]

4. Zheng, J. SARS-CoV-2: An emerging coronavirus that causes a global threat. Int. J. Biol. Sci. 2020, 16, 1678-1685. [CrossRef] [PubMed]

5. Oh, K.K.; Adnan, M.; Cho, D.H. Network pharmacology approach to decipher signaling pathways associated with target proteins of NSAIDs against COVID-19. Sci. Rep. 2021, 11, 9606. [CrossRef] [PubMed]

6. Oh, K.K.; Adnan, M.; Cho, D.H. Drug-repurposing against COVID-19 by targeting a key signaling pathway: An in silico study. Med. Hypotheses 2021, 155, 110656. [CrossRef] [PubMed]

7. WHO Declares Coronavirus Outbreak a Global Health Emergency—STAT; WHO: Geneva, Switzerland, 2020.

8. COVID-19 Map-Johns Hopkins Coronavirus Resource Center. Available online: https://coronavirus.jhu.edu/map.html (accessed on 20 December 2021).

9. Huang, C.; Wang, Y.; Li, X.; Ren, L.; Zhao, J.; Hu, Y.; Zhang, L.; Fan, G.; Xu, J.; Gu, X.; et al. Clinical features of patients infected with 2019 novel coronavirus in Wuhan, China. Lancet 2020, 395, 497-506. [CrossRef]

10. Speth, M.M.; Singer-Cornelius, T.; Oberle, M.; Gengler, I.; Brockmeier, S.J.; Sedaghat, A.R. Olfactory Dysfunction and Sinonasal Symptomatology in COVID-19: Prevalence, Severity, Timing, and Associated Characteristics. Otolaryngol. Head Neck Surg. 2020, 163, 114-120. [CrossRef] [PubMed]

11. Oran, D.P.; Topol, E.J. Prevalence of Asymptomatic SARS-CoV-2 Infection. Ann. Intern. Med. 2020, 173, 362-367. [CrossRef] 
12. Azman, S.; Sekar, M.; Wahidin, S.; Gan, S.H.; Vaijanathappa, J.; Bonam, S.R.; Alvala, M.; Lum, P.T.; Thakur, V.; Beladiya, J.V.; et al. Embelin Alleviates Severe Airway Inflammation in OVA-LPS-Induced Rat Model of Allergic Asthma. J. Asthma Allergy 2021, 14, 1511-1525. [CrossRef]

13. Sanyaolu, A.; Okorie, C.; Marinkovic, A.; Patidar, R.; Younis, K.; Desai, P.; Hosein, Z.; Padda, I.; Mangat, J.; Altaf, M. Comorbidity and its Impact on Patients with COVID-19. SN Compr. Clin. Med. 2020, 2, 1069-1076. [CrossRef] [PubMed]

14. Gordon, D.E.; Jang, G.M.; Bouhaddou, M.; Xu, J.; Obernier, K.; White, K.M.; O’Meara, M.J.; Rezelj, V.V.; Guo, J.Z.; Swaney, D.L.; et al. A SARS-CoV-2 protein interaction map reveals targets for drug repurposing. Nature 2020, 583, 459-468. [CrossRef] [PubMed]

15. Adnan, M.; Chy, M.N.U.; Kamal, A.T.M.M.; Chowdhury, K.A.A.; Rahman, M.A.; Reza, A.S.M.A.; Moniruzzaman, M.; Rony, S.R.; Nasrin, M.S.; Azad, M.O.K.; et al. Intervention in Neuropsychiatric Disorders by Suppressing Inflammatory and Oxidative Stress Signal and Exploration of In Silico Studies for Potential Lead Compounds from Holigarna caustica (Dennst.) Oken leaves. Biomolecules 2020, 10, 561. [CrossRef] [PubMed]

16. Islam, M.T.; Sarkar, C.; El-Kersh, D.M.; Jamaddar, S.; Uddin, S.J.; Shilpi, J.A.; Mubarak, M.S. Natural products and their derivatives against coronavirus: A review of the non-clinical and pre-clinical data. Phyther. Res. 2020, 34, 2471-2492. [CrossRef] [PubMed]

17. Desborough, M.J.R.; Keeling, D.M. The aspirin story-From willow to wonder drug. Br. J. Haematol. 2017, 177, 674-683. [CrossRef] [PubMed]

18. Liu, N.Q.; Schuehly, W.; von Freyhold, M.; Van der Kooy, F. A novel purification method of artemisinin from Artemisia annua. Ind. Crops Prod. 2011, 34, 1084-1088. [CrossRef]

19. Rao, K.V. Taxol and Related Taxanes. I. Taxanes of Taxus brevifolia Bark. Pharm. Res. An Off. J. Am. Assoc. Pharm. Sci. 1993, 10, 521-524. [CrossRef]

20. Chen, Z.; Nakamura, T. Statistical evidence for the usefulness of Chinese medicine in the treatment of SARS. Phyther. Res. 2004, 18, 592-594. [CrossRef] [PubMed]

21. Zhang, D.; Zhang, X.; Peng, B.; Deng, S.; Wang, Y.; Yang, L.; Zhang, K.; Ling, C.; Wu, K. Network pharmacology suggests biochemical rationale for treating COVID-19 symptoms with a Traditional Chinese Medicine. Commun. Biol. 2020, 3 , 466. [CrossRef] [PubMed]

22. Wee, J.J.; Park, K.M.; Chung, A.S. Biological activities of ginseng and its application to human health. In Herbal Medicine: Biomolecular and Clinical Aspects, 2nd ed.; CRC Press: Boca Raton, FL, USA, 2011; pp. 157-174. ISBN 9781439807163.

23. Lee, J.S.; Ko, E.J.; Hwang, H.S.; Lee, Y.N.; Kwon, Y.M.; Kim, M.C.; Kang, S.M. Antiviral activity of ginseng extract against respiratory syncytial virus infection. Int. J. Mol. Med. 2014, 34, 183-190. [CrossRef] [PubMed]

24. Nguyen, N.H.; Nguyen, C.T. Pharmacological effects of ginseng on infectious diseases. Inflammopharmacology 2019, 27, 871-883. [CrossRef]

25. Leung, K.W.; Wong, A.S. Pharmacology of ginsenosides: A literature review. Chin. Med. 2010, 5, 20. [CrossRef] [PubMed]

26. Han, M.; Fang, X.-L. Difference in oral absorption of ginsenoside Rg 1 between in vitro and in vivo models. Acta Pharmacol. Sin. 2006, 27, 499-505. [CrossRef] [PubMed]

27. Zhang, B.; Zhu, X.M.; Hu, J.N.; Ye, H.; Luo, T.; Liu, X.R.; Li, H.Y.; Li, W.; Zheng, Y.N.; Deng, Z.Y. Absorption mechanism of ginsenoside compound $\mathrm{K}$ and its butyl and octyl ester prodrugs in caco-2 cells. J. Agric. Food Chem. 2012, 60, 10278-10284. [CrossRef] [PubMed]

28. Bennion, B.J.; Be, N.A.; Mcnerney, M.W.; Lao, V.; Carlson, E.M.; Valdez, C.A.; Malfatti, M.A.; Enright, H.A.; Nguyen, T.H.; Lightstone, F.C.; et al. Predicting a Drug's Membrane Permeability: A Computational Model Validated with In Vitro Permeability Assay Data. J. Phys. Chem. B 2017, 121, 5228-5237. [CrossRef] [PubMed]

29. Oh, K.; Adnan, M.; Cho, D. Uncovering Mechanisms of Zanthoxylum piperitum Fruits for the Alleviation of Rheumatoid Arthritis Based on Network Pharmacology. Biology 2021, 10, 703. [CrossRef] [PubMed]

30. Hopkins, A.L. Network pharmacology: The next paradigm in drug discovery. Nat. Chem. Biol. 2008, 4, 682-690. [CrossRef] [PubMed]

31. Xu, L.; Zhang, Y.; Zhang, P.; Dai, X.; Gao, Y.; Lv, Y.; Qin, S.; Xu, F. Integrated Metabolomics and Network Pharmacology Strategy-Driven Active Traditional Chinese Medicine Ingredients Discovery for the Alleviation of Cisplatin Nephrotoxicity. Chem. Res. Toxicol. 2019, 32, 2411-2421. [CrossRef] [PubMed]

32. Chowdhury, H.U.; Adnan, M.; Oh, K.K.; Cho, D.H. Decrypting molecular mechanism insight of Phyllanthus emblica L. fruit in the treatment of type 2 diabetes mellitus by network pharmacology. Phytomedicine Plus 2021, 1, 100144. [CrossRef]

33. Tao, L.; Lowe, A.; Wang, G.; Dozmorov, I.; Chang, T.; Yan, N.; Reese, T. Metabolic Control of Viral Infection through PPAR- $\alpha$ Regulation of STING Signaling. bioRxiv 2019, 731208. [CrossRef]

34. Shi, W.; Hou, X.; Li, X.; Peng, H.; Shi, M.; Jiang, Q.; Liu, X.; Ji, Y.; Yao, Y.; He, C.; et al. Differential gene expressions of the MAPK signaling pathway in enterovirus 71-infected rhabdomyosarcoma cells. Brazilian J. Infect. Dis. 2013, 17, 410-417. [CrossRef] [PubMed]

35. Grimes, J.M.; Grimes, K.V. p38 MAPK inhibition: A promising therapeutic approach for COVID-19. J. Mol. Cell. Cardiol. 2020, 144, 63-65. [CrossRef] [PubMed]

36. Ho, J.; Moyes, D.L.; Tavassoli, M.; Naglik, J.R. The Role of ErbB Receptors in Infection. Trends Microbiol. 2017, 25, 942-952. [CrossRef] [PubMed] 
37. Menzo, S.; Clementi, M.; Alfani, E.; Bagnarelli, P.; Iacovacci, S.; Manzin, A.; Dandri, M.; Natoli, G.; Levrero, M.; Carloni, G. Trans-Activation of Epidermal Growth Factor Receptor Gene by the Hepatitis B Virus X-Gene Product. Virology 1993, 196, 878-882. [CrossRef] [PubMed]

38. Miyaki, M.; Sato, C.; Sakai, K.; Konishi, M.; Tanaka, K.; Muraoka, M.; Kikuchi-Yanoshita, R.; Nadaoka, Y.; Kanda, H.; Kitagawa, T. Malignant transformation and EGFR activation of immortalized mouse liver epithelial cells caused by HBV enhancer- $X$ from a human hepatocellular carcinoma. Int. J. Cancer 2000, 85, 518-522. [CrossRef]

39. Battagello, D.S.; Dragunas, G.; Klein, M.O.; Ayub, A.L.P.; Velloso, F.J.; Correa, R.G. Unpuzzling COVID-19: Tissue-related signaling pathways associated with SARS-CoV-2 infection and transmission. Clin. Sci. 2020, 134, 2137-2160. [CrossRef]

40. Moreno-Fernandez, M.E.; Rueda, C.M.; Velilla, P.A.; Rugeles, M.T.; Chougnet, C.A. cAMP during HIV infection: Friend or foe? AIDS Res. Hum. Retrovir. 2012, 28, 49-53. [CrossRef]

41. Coperchini, F.; Chiovato, L.; Croce, L.; Magri, F.; Rotondi, M. The cytokine storm in COVID-19: An overview of the involvement of the chemokine/chemokine-receptor system. Cytokine Growth Factor Rev. 2020, 53, 25-32. [CrossRef] [PubMed]

42. DeDiego, M.L.; Nieto-Torres, J.L.; Regla-Nava, J.A.; Jimenez-Guardeno, J.M.; Fernandez-Delgado, R.; Fett, C.; Castano-Rodriguez, C.; Perlman, S.; Enjuanes, L. Inhibition of NF- B-Mediated Inflammation in Severe Acute Respiratory Syndrome CoronavirusInfected Mice Increases Survival. J. Virol. 2014, 88, 913-924. [CrossRef]

43. Codo, A.C.; Davanzo, G.G.; de Monteiro, L.B.; de Souza, G.F.; Muraro, S.P.; Virgilio-da-Silva, J.V.; Prodonoff, J.S.; Carregari, V.C.; de Biagi Junior, C.A.O.; Crunfli, F.; et al. Elevated Glucose Levels Favor SARS-CoV-2 Infection and Monocyte Response through a HIF-1 $\alpha /$ Glycolysis-Dependent Axis. Cell Metab. 2020, 32, 437-446. [CrossRef]

44. Sullivan, J.A.; Kim, E.H.; Plisch, E.H.; Suresh, M. FOXO3 Regulates the CD8 T Cell Response to a Chronic Viral Infection. J. Virol. 2012, 86, 9025-9034. [CrossRef] [PubMed]

45. Abu-Farha, M.; Thanaraj, T.A.; Qaddoumi, M.G.; Hashem, A.; Abubaker, J.; Al-Mulla, F. The role of lipid metabolism in COVID-19 virus infection and as a drug target. Int. J. Mol. Sci. 2020, 21, 3544. [CrossRef] [PubMed]

46. Hannun, Y.A.; Obeid, L.M. Sphingolipids and their metabolism in physiology and disease. Nat. Rev. Mol. Cell Biol. 2018, 19, 175-191. [CrossRef] [PubMed]

47. Huwiler, A.; Zangemeister-Wittke, U. The sphingosine 1-phosphate receptor modulator fingolimod as a therapeutic agent: Recent findings and new perspectives. Pharmacol. Ther. 2018, 185, 34-49. [CrossRef] [PubMed]

48. Lythgoe, M.P.; Middleton, P. Ongoing Clinical Trials for the Management of the COVID-19 Pandemic. Trends Pharmacol. Sci. 2020, 41, 363-382. [CrossRef]

49. Ji, W.-T.; Liu, H. PI3K-Akt Signaling and Viral Infection. Recent Pat. Biotechnol. 2008, 2, 218-226. [CrossRef] [PubMed]

50. Mizutani, T.; Fukushi, S.; Saijo, M.; Kurane, I.; Morikawa, S. Importance of Akt signaling pathway for apoptosis in SARS-CoVinfected Vero E6 cells. Virology 2004, 327, 169-174. [CrossRef]

51. Silwal, P.; Kim, J.K.; Yuk, J.M.; Jo, E.K. AMP-Activated protein kinase and host defense against infection. Int. J. Mol. Sci. 2018, 19, 3495. [CrossRef]

52. van Zuylen, W.J.; Rawlinson, W.D.; Ford, C.E. The Wnt pathway: A key network in cell signalling dysregulated by viruses. Rev. Med. Virol. 2016, 26, 340-355. [CrossRef] [PubMed]

53. Onofrio, L.; Caraglia, M.; Facchini, G.; Margherita, V.; De Placido, S.; Buonerba, C. Toll-like receptors and COVID-19: A two-faced story with an exciting ending. Futur. Sci. OA 2020, 6, FSO605. [CrossRef]

54. Shaw, P.J.; Lamkanfi, M.; Kanneganti, T.D. NOD-like receptor (NLR) signaling beyond the inflammasome. Eur. J. Immunol. 2010, 40, 624-627. [CrossRef] [PubMed]

55. Lei, X.; Dong, X.; Ma, R.; Wang, W.; Xiao, X.; Tian, Z.; Wang, C.; Wang, Y.; Li, L.; Ren, L.; et al. Activation and evasion of type I interferon responses by SARS-CoV-2. Nat. Commun. 2020, 11, 1-12. [CrossRef] [PubMed]

56. Goker Bagca, B.; Biray Avci, C. The potential of JAK/STAT pathway inhibition by ruxolitinib in the treatment of COVID-19. Cytokine Growth Factor Rev. 2020, 54, 51-61. [CrossRef] [PubMed]

57. Pacha, O.; Sallman, M.A.; Evans, S.E. COVID-19: A case for inhibiting IL-17? Nat. Rev. Immunol. 2020, 20, 345-346. [CrossRef] [PubMed]

58. Chen, Z.; John Wherry, E. T cell responses in patients with COVID-19. Nat. Rev. Immunol. 2020, 20, 529-536. [CrossRef] [PubMed]

59. Purtha, W.E.; Chachu, K.A.; Virgin, H.W.; Diamond, M.S. Early B-Cell Activation after West Nile Virus Infection Requires Alpha/Beta Interferon but Not Antigen Receptor Signaling. J. Virol. 2008, 82, 10964-10974. [CrossRef] [PubMed]

60. Catanzaro, M.; Fagiani, F.; Racchi, M.; Corsini, E.; Govoni, S.; Lanni, C. Immune response in COVID-19: Addressing a pharmacological challenge by targeting pathways triggered by SARS-CoV-2. Signal Transduct. Target. Ther. 2020, 5, 1-10. [CrossRef] [PubMed]

61. Wood, H. New insights into the neurological effects of COVID-19. Nat. Rev. Neurol. 2020, 16, 403. [CrossRef] [PubMed]

62. Barabás, K.; Szabó-Meleg, E.; Ábrahám, I.M. Effect of inflammation on female gonadotropin-releasing hormone (GnRH) neurons: Mechanisms and consequences. Int. J. Mol. Sci. 2020, 21, 529. [CrossRef]

63. Acharya, S.; Fernando, J.J.R.; Gama, R. Gynaecomastia, hyperprolactinaemia and HIV infection. Ann. Clin. Biochem. 2005, 42, 301-303. [CrossRef]

64. Borba, V.V.; Zandman-Goddard, G.; Shoenfeld, Y. Prolactin and autoimmunity: The hormone as an inflammatory cytokine. Best Pract. Res. Clin. Endocrinol. Metab. 2019, 33, 101324. [CrossRef] [PubMed] 
65. Mancuso, P. Pulmonary Physiology and Pathophysiology in Obesity Obesity and lung inflammation. J. Appl. Physiol. 2010, 108, 722-728. [CrossRef] [PubMed]

66. Khan, M.A.-A.-K.; Islam, A.B.M.M.K. SARS-CoV-2 proteins exploit host's genetic and epigenetic mediators for the annexation of key host signaling pathways that confers its immune evasion and disease pathophysiology. bioRxiv 2020, 050260. [CrossRef]

67. Hudson, B.I.; Lippman, M.E. Targeting RAGE Signaling in Inflammatory Disease. Annu. Rev. Med. 2018, 69, 349-364. [CrossRef] [PubMed]

68. Reyes, V.E.; Peniche, A.G. Helicobacter pylori deregulates T and B cell signaling to trigger immune evasion. In Current Topics in Microbiology and Immunology; Springer: Berlin/Heidelberg, Germany, 2019; Volume 421, pp. 229-265.

69. Liu, T.; Zhang, L.; Joo, D.; Sun, S.C. NF-kB signaling in inflammation. Signal Transduct. Target. Ther. 2017, 2, 17023. [CrossRef]

70. Tanaka, N. The anti-apoptotic protein MCL1, a novel target of lung cancer therapy. J. Cancer Treat. Diagn. 2017, 2, 54-58. [CrossRef]

71. Chen, L.; Li, X.; Cheng, M.; Wang, S.; Zheng, Q.; Liu, Q. Iso-pencillixanthone A from a marine-derived fungus reverses multidrug resistance in cervical cancer cells through down-regulating P-gp and re-activating apoptosis. RSC Adv. 2018, 8, 41192-41206. [CrossRef]

72. Turkia, M. COVID-19 as an Endothelial Disease and Its Relationship to Vascular Endothelial Growth Factor (VEGF) and Iodide. SSRN Electron. J. 2020, 1-14. [CrossRef]

73. Rahmati, M. Cytokine-targeted therapy in severely ill COVID-19 patients: Options and cautions. Eurasian J. Med. Oncol. 2020, 4 , 179-181. [CrossRef] 